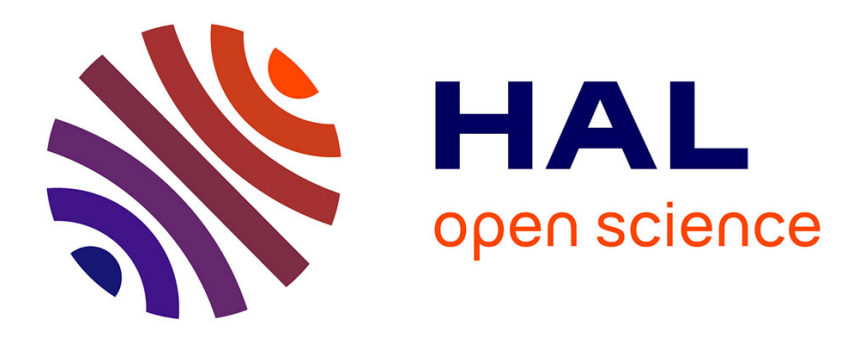

\title{
Internal wave generation in uniformly stratified fluids. Part 2. Moving point sources \\ Bruno Voisin
}

\section{To cite this version:}

Bruno Voisin. Internal wave generation in uniformly stratified fluids. Part 2. Moving point sources. Journal of Fluid Mechanics, 1994, 261, pp.333-374. 10.1017/S0022112094000364 hal-01943517

\section{HAL Id: hal-01943517 https://hal.science/hal-01943517}

Submitted on 8 Dec 2018

HAL is a multi-disciplinary open access archive for the deposit and dissemination of scientific research documents, whether they are published or not. The documents may come from teaching and research institutions in France or abroad, or from public or private research centers.
L'archive ouverte pluridisciplinaire HAL, est destinée au dépôt et à la diffusion de documents scientifiques de niveau recherche, publiés ou non, émanant des établissements d'enseignement et de recherche français ou étrangers, des laboratoires publics ou privés. 


\title{
Internal wave generation in uniformly stratified fluids. Part 2. Moving point sources
}

\author{
By BRUNO VOISIN \\ Laboratoire des Ecoulements Géophysiques et Industriels, Institut de Mécanique de Grenoble, \\ CNRS - UJF - INPG, BP 53X, 38041 Grenoble Cedex, France
}

(Received 29 September 1992 and in revised form 27 May 1993)

The Green's function method is applied to the generation of internal gravity waves by a moving point mass source. Arbitrary motion of a source of arbitrary time dependence is treated using the impulsive Green's function, while 'classical' approaches of uniform motion of a steady or oscillatory source are recovered using the monochromatic Green's function. Waves have locally the structure of impulsive waves, emitted at the retarded time $t_{r}$ and having propagated with the group velocity; at each position and time an implicit equation defines $t_{r}$, in terms of which the waves are written. A source both oscillating and moving generates two systems of waves, with respectively positive and negative frequencies, and when oscillations vanish these systems merge into one.

Three particular cases are considered: the uniform horizontal and vertical motions of a steady source, and the uniform horizontal motion of an oscillatory source. Waves spread downstream of the steady source. For the oscillatory source they can extend both upstream and downstream, depending on the ratio of the source frequency to the buoyancy frequency, and are contained inside conical wavefronts, parts of which are caustics. For horizontal motion, moreover, the steady analysis (based on the monochromatic Green's function) reveals the presence of two insignificant contributions overlooked by the unsteady analysis (based on the impulsive Green's function), but which for an extended source may become of the same order as the main contribution. Among those is an upstream columnar disturbance associated with blocking.

\section{Introduction}

Waves generated by bodies moving in stratified fluids, or by obstacles fixed in stratified flows, are certainly one of the most striking forms of internal gravity waves. In the atmosphere, characteristic cloud patterns in the lee of mountains or hills over which stratified winds blow reveal the presence of 'lee waves', of primary importance for e.g. meteorology, aerial navigation, pollution problems and gliding (Miles 1969c). In the ocean, ships moving in strongly stratified coastal waters undergo significant drag attributable to internal wave generation, and originally ascribed to the attachment of 'dead water' (Ekman 1904).

Acoustic (Morse \& Feshbach 1953, pp. 841-842) or electromagnetic (Landau \& Lifchitz 1970, §63) waves radiated by acoustic or electromagnetic moving sources are well-known. The clue to their understanding is the so-called retarded time $t_{r}$, which 
represents the time when the waves received at point $\boldsymbol{r}$ and time $t$ were emitted, and which satisfies, for source path $\boldsymbol{r}_{0}(t)$, the implicit equation

$$
t-t_{r}=\frac{\left|\boldsymbol{r}-\boldsymbol{r}_{0}\left(t_{r}\right)\right|}{c},
$$

expressing propagation at the sound or light velocity $c$. Once $t_{r}$ is known the determination of the waves is straightforward, and results in the so-called LiénardWiechert potentials.

For internal waves, dispersion and anisotropy imply the separation, and dependence on position and time, of the velocities $c_{\phi}$ of phase propagation and $c_{g}$ of energy propagation. Equation (1.1) becomes

$$
t-t_{r}=\frac{\left|\boldsymbol{r}-\boldsymbol{r}_{0}\left(t_{r}\right)\right|}{c_{g}},
$$

with $c_{g}$ an unspecified function of $\boldsymbol{r}, t$ and $t_{r}$. Little information on the waves is then obtained in this way, save a parametric equation of surfaces of constant phase deduced by Stevenson (1973), Peat \& Stevenson $(1975,1976)$ and Woodhead (1983) from the combination of (1.2) with the Doppler and dispersion relations.

This paper shows how the use of Green's functions overcomes the difficulty, leading to a complete solution to the problem of internal wave radiation by moving sources, formulated in terms of just the retarded time. In so doing it continues the development of the Green's function theory initiated in Voisin $(1991 \mathrm{~b}$, hereafter referred to as I). Indeed, Lighthill's (1967, 1978, §4.12) analysis of the generation of anisotropic dispersive waves already deals with uniformly moving sources, building the wave field in Fourier space and time from a superposition of wavepackets of various frequencies and wavenumbers. On the other hand, the Green's function method builds the wave field in real space and time, for arbitrarily moving sources, from a superposition of transients emitted at various times by the various points of the source. Both theories are, thus, hoped to provide complementary insights into the problem under consideration.

Throughout the paper it is assumed that waves are linear and three-dimensional, that the fluid is inviscid, incompressible, unbounded, uniformly stratified (i.e. of constant buoyancy frequency) and non-rotating, and that the Boussinesq approximation holds. First $\S 2$ reviews the extensive literature on the generation of internal waves by moving bodies. Section 3 applies the Green's function formalism to arbitrary motion of a point source of arbitrary time dependence, while in $\S 4$ it is specialized to uniform motion of a steady or oscillating point source. Then, using both approaches, three cases of practical interest are considered: the uniform horizontal motion of a steady source ( $(5)$, with applications to lee waves (Miles $1969 c$ ), the uniform vertical motion of a steady source $(\S 6$ ), with applications to thermals (Warren 1960), and the uniform horizontal motion of an oscillating source $(\S 7)$, with applications to stratified wakes (Gilreath \& Brandt 1985).

\section{Bibliographical review}

Studies of the generation of internal waves by bodies moving in unbounded uniformly stratified fluids can be classified into (i) theoretical investigations of moving point sources, (ii) theoretical investigations of moving extended sources, (iii) experimental or numerical investigations. Here we review all three approaches successively, 
restricting our attention to three-dimensional theoretical work and to both two- and three-dimensional experimental or numerical work.

\subsection{Point sources}

Early analyses of moving point sources rely on essentially the same 'integral' method, in which the waves are calculated in Fourier space and evaluated asymptotically by stationary phase or steepest descent arguments. For uniform horizontal motion of a steady source this procedure has been introduced by Wurtele (1957) and Wu (1965), with errors, and has been applied by Crapper (1959) far from the source but near to its path and by Miles (1971), Dokuchaev \& Dolina (1977), Sturova (1978) and Janowitz (1984) throughout the far field, while Trubnikov (1959) derived only the Fourier integral form of the waves and Sturova (1974) evaluated it numerically. Uniform vertical motion of a steady source (Warren 1960; Grigor'ev \& Dokuchaev 1970) and uniform horizontal motion of an oscillating source (Rehm \& Radt 1975) have been treated in the same way, and for arbitrary motion the introduction of Fourier-Laplace transforms has led to an expression of the waves as an integral over time (Sturova 1980; Chashechkin \& Makarov 1984).

A more systematic procedure was offered by Lighthill's $(1967,1978, \S 4.12)$ theory of the generation of anisotropic dispersive waves. First, the combination of the Doppler and dispersion relations defines a 'wavenumber' surface, of which surfaces of constant phase are reciprocal polars. These have been studied for horizontal (Redekopp 1975; Rehm \& Radt 1975; Lighthill 1978, §4.12) and vertical (Mowbray \& Rarity 1967; Lighthill 1967, 1978, \$4.12) motions of a steady source, and for horizontal (Redekopp 1975; Rehm \& Radt 1975) and vertical (Stevenson 1969; Subba Rao \& Prabhakara Rao 1971) motions of an oscillating source. The wave amplitude follows, similarly, from the curvature of the wavenumber surface. It has been calculated by Makarov \& Chashechkin $(1981,1982)$ for horizontal and vertical motions of a steady source. If, moreover, the retarded time equation is added to the Doppler and dispersion relations, arbitrary motion can be considered. On these grounds, Stevenson (1973), Peat \& Stevenson $(1975,1976)$ and Woodhead (1983) have built a parametric construction of surfaces of constant phase, and have applied it to various accelerated or curved motions.

A third angle of attack is to write the waves as four-dimensional Fourier integrals over wavenumbers and frequency, and to deduce therefrom both the exact form of the waves in particular places and the total radiated energy. It has been introduced by Gorodtsov \& Teodorovich $(1980,1981)$ for uniform horizontal motion, by Gorodtsov (1980) for uniform vertical motion, and by Gorodtsov \& Teodorovich (1983) for some periodic motions.

\subsection{Extended sources}

The motion at constant speed $U$ of a body of transverse radius $a$ in a fluid of buoyancy frequency $N$ is ruled by the internal Froude number $\mathrm{Fr}=U / \mathrm{Na}$, representing the ratio of inertial forces to buoyancy forces.

For $F r \ll 1$ (strong stratification), the forcing velocity $U$ is small compared with $\mathrm{Na}$, and linearization around the state of rest of both the fluid and the body is appropriate. This problem has been solved by Grimshaw (1969) and Sarma \& Krishna (1972) for impulsively started motion of a sphere, and by Vladimirov \& Il'in (1991) for arbitrary body shape. The velocity field comprises two terms: transient waves generated by the impulsive start, and the steady stratified flow around the body. For horizontal motion, because of the strength of the stratification, this flow is horizontal and irrotational, 
and reduces to just the leading-order flow derived by Drazin (1961) and Brighton (1978) from an expansion of the nonlinear equations of motion in powers of $\mathrm{Fr}^{2}$.

For $F r \gg 1$ (weak stratification), the radius $a$ of the body is small compared with the distance $U / N$ for stratification effects to be significant. Thus, consistently with the method of matched asymptotic expansions (Murdock 1977; Baines \& Grimshaw 1979) or the triple-deck analysis (Sykes 1978), the fluid must be separated in two zones (Miles \& Huppert 1969; Miles 1969a, 1971). Near to the body, at distances of order $a$, the flow is weakly affected by the stratification; to leading order in $1 / F r$ it becomes a three-dimensional irrotational flow, higher orders following from a perturbation procedure developed by Hawthorne \& Martin (1955) for a sphere and by Drazin (1961) for arbitrary body shape. Far from the body, at distances of order $U / N$, internal waves are observed with velocities small compared with $U$; linearization is again appropriate, around a reference state in which the fluid is at rest and the body moves. Matching those inner and outer solutions in the intermediate zone $a \ll R \ll U / N$, with $R$ the distance from the body, legitimates using for the waves the same representation of the body as for homogeneous flow; once this is known, results of Gorodtsov $(1981,1991)$ and Gorodtsov \& Teodorovich (1982) describe the radiated energy.

Details of the wave field depend then on the aspect ratio $\varepsilon=l / a$, with $2 l$ the length of the body. For $\varepsilon=O(1)$, the literature considers either a dipole (see $\S 2.1$ ) or a surface distribution of sources and sinks (Gorodtsov \& Teodorovich 1982), which are respectively far-field $(R \gg a)$ and exact representations of the body in homogeneous flow. For $\varepsilon \gg 1$ classical slender-body theory is relevant, as is, for surface-mounted obstacles of height $h$ and horizontal dimension $2 l$ satisfying $U / N h \gg 1$ and $l / h \gg 1$, flat-body theory. Both of them have been applied by Warren (1960) to vertical motion and by Crapper $(1959,1962)$, Janowitz (1984) and Umeki \& Kambe (1989) to horizontal motion. When, moreover, for horizontal motion, the Froude number $U / N l$ based on streamwise length is small, the hydrostatic approximation holds (Blumen \& McGregor 1976; Smith 1980).

For $F r=O(1)$ the flow around the body interacts strongly with the wave field, vitiating the preceding approaches. There is, nonetheless, both experimental (Hunt \& Snyd.r 1980) and numerical (Hanazaki 1988; Smolarkiewicz \& Rotunno 1989; Suzuki \& Kuwahara 1992) evidence that the range of validity of linear theories is remarkably large, extending for small- $F r$ theories up to, say, 0.1 , and for large- $F r$ theories down to, say, 2. Anticipating this, for flat obstacles, Scorer (1956), Smith $(1980,1988,1989)$ and Phillips (1984) have applied the large-Fr linearization in the whole of the fluid, while Chashechkin (1989) proposed, whatever $F r$, to replace the body by a discrete distribution of sources and sinks of position and strength fixed by experiment.

\subsection{Experiments and numerical simulations}

Experiments on and simulations of the motion of bodies in stratified fluids are numerous. Here we only consider those focused on waves. For complementary reviews focused on flow effects, the reader is referred to Boyer et al. (1989) in two dimensions and Lin et al. (1992) in three dimensions, and for more emphasis on wake phenomena to Sysoeva \& Chashechkin (1991) and Chomaz, Bonneton \& Hopfinger (1993).

Experiments have involved a wide range of bodies and motions. This includes: a cylinder, in uniform horizontal (Stevenson, Chang \& Laws 1979; Stevenson, Woodhead \& Kanellopulos 1983; Belotserkovskii et al. 1984; Aksenov et al. 1989), vertical 


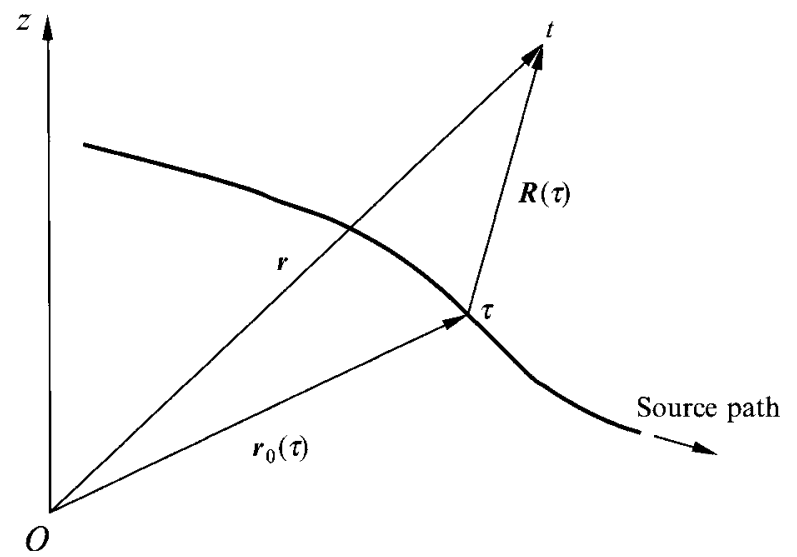

FIGURE 1. Geometry for internal wave radiation by an arbitrarily moving source [adapted from Stevenson 1973].

(Stevenson et al. 1983; Gärtner 1983a, b; Gärtner, Wernekinck \& Merzkirch 1986) or inclined (Stevenson 1968) motion, or in uniform horizontal or inclined motion with oscillations (Stevenson \& Thomas 1969), or in accelerated or curved motion (Stevenson 1973; Peat \& Stevenson 1975, 1976; Woodhead 1983); a sphere, in uniform horizontal (Peat \& Stevenson 1975; Makarov \& Chashechkin 1981, 1982; Chashechkin 1989; Hopfinger et al. 1991; Bonneton, Chomaz \& Hopfinger 1993), vertical (Mowbray \& Rarity 1967) or inclined (Peat \& Stevenson 1975) motion, or in uniform vertical (Stevenson 1969) or horizontal or inclined (Peat \& Stevenson 1975) motion with oscillations; a slender ellipsoid (Gilreath \& Brandt 1985), a triangular ridge of finite width (Castro, Snyder \& Marsh 1983; Castro 1987), and two-dimensional obstacles (Baines \& Hoinka 1985), all of which in uniform horizontal motion.

Numerical simulations are comparatively few. They deal with uniform horizontal motion of a cylinder (Belotserkovskii et al. 1984), a sphere (Hanazaki 1988), rectangular (Foldvik \& Wurtele 1967) or semi-elliptical (Haussling 1977) ridges of infinite width, two- (Clark \& Peltier 1977; Klemp \& Lilly 1978; Peltier \& Clark 1979, 1983) or three- (Smolarkiewicz \& Rotunno 1989; Rotunno \& Smolarkiewicz 1991; Suzuki \& Kuwahara 1992) dimensional bell-shaped obstacles, and a parallelopiped (Sharman $\&$ Wurtele 1983).

\section{Internal waves generated by an arbitrarily moving source}

At time $t=0$, the monopolar point mass source

$$
m(\boldsymbol{r}, t)=H(t) m_{0}(t) \mathrm{e}^{\mathrm{i} \omega_{0} t} \delta\left[\boldsymbol{r}-\boldsymbol{r}_{0}(t)\right],
$$

situated at point $O$, starts to move along the path $\boldsymbol{r}_{0}(t)$ while its strength starts to oscillate with frequency $\omega_{0}$ and otherwise to undergo non-periodic variations $m_{0}(t)$, as sketched in figure 1 . Here $\boldsymbol{r}=[x, y, z]$ denotes position, of horizontal and vertical components $\boldsymbol{r}_{h}=[x, y, 0]$ and $z$, and $H$ and $\delta$ are the Heaviside step and Dirac delta functions, respectively.

We assume that the fluid is incompressible, unbounded and uniformly stratified (i.e. of constant buoyancy frequency $N$ ), that wave amplitudes are small and that the Boussinesq approximation is valid. Then the fluid velocity $v(r, t)$ and pressure 
perturbation $P(r, t)$ can be written

$$
\boldsymbol{v}(\boldsymbol{r}, t)=\left(\frac{\partial^{2}}{\partial t^{2}} \nabla+N^{2} \nabla_{h}\right) \psi(\boldsymbol{r}, t), \quad P(\boldsymbol{r}, t)=-\rho_{0}\left(\frac{\partial^{2}}{\partial t^{2}}+N^{2}\right) \frac{\partial}{\partial t} \psi(\boldsymbol{r}, t),
$$

where $\rho_{0}$ is the undisturbed density and $\psi(\boldsymbol{r}, t)$ is an 'internal potential' satisfying

$$
\left(\frac{\partial^{2}}{\partial t^{2}} \nabla^{2}+N^{2} \nabla_{h}^{2}\right) \psi(\boldsymbol{r}, t)=m(\boldsymbol{r}, t)
$$

with $\nabla=[\partial / \partial x, \partial / \partial y, \partial / \partial z]$ and $\nabla_{h}=[\partial / \partial x, \partial / \partial y, 0]$. In terms of the causal Green's function $G(\boldsymbol{r}, t)$ of equation (3.3), defined by

$$
\left(\frac{\partial^{2}}{\partial t^{2}} \nabla^{2}+N^{2} \nabla_{h}^{2}\right) G(\boldsymbol{r}, t)=\delta(\boldsymbol{r}) \delta(t),\left.\quad G(\boldsymbol{r}, t)\right|_{t<0}=0,
$$

the internal potential is given by the convolution integral

$$
\psi(\boldsymbol{r}, t)=\int \mathrm{d} \tau \int \mathrm{d}^{3} r^{\prime} m\left(\boldsymbol{r}^{\prime}, \tau\right) G\left(\boldsymbol{r}-\boldsymbol{r}^{\prime}, t-\tau\right)
$$

which for the source (3.1) becomes

$$
\psi(\boldsymbol{r}, t)=H(t) \int_{0}^{t} m_{0}(\tau) \mathrm{e}^{\mathrm{i} \omega_{0} \tau} G\left[\boldsymbol{r}-\boldsymbol{r}_{0}(\tau), t-\tau\right] \mathrm{d} \tau
$$

Internal waves are observed only for $t>0$, as reflected by the factor $H(t)$ which is suppressed in the following. Then they result from a superposition of impulses generated at each time $\tau$ and point $\boldsymbol{r}_{0}(\tau)$ of the path of the source, as pointed out by Bretherton (1967, p. 559) and Miles (1969b, p. 438).

Similar integrals govern the radiation of acoustic and electromagnetic waves (Morse \& Feshbach 1953, pp. 841-842; Landau \& Lifchitz 1970, §63). For them, the Green's function contains a Dirac function $\delta(r-c t)$, so that each $\boldsymbol{r}$ receives at each $t$ an impulse emitted at the retarded time $\tau_{r}$, defined by (1.1) with $t_{r}$ replaced by $\tau_{r}$. For internal waves there is no such Dirac function in the Green's function, but, from group velocity arguments, the retarded time still exists. To distinguish this particular $\tau$ in (3.6) we shall resort to an asymptotic procedure, based on expansion (6.15) in I, valid for $N t \gg 1$,

$$
G(\boldsymbol{r}, t) \sim-\frac{1}{(2 \pi)^{\frac{3}{2}} N r_{h}}\left[\frac{\cos \left(N t \frac{|z|}{r}-\frac{\pi}{4}\right)}{\left(N t \frac{|z|}{r}\right)^{\frac{1}{2}}}+\frac{\sin \left(N t-\frac{\pi}{4}\right)}{(N t)^{\frac{1}{2}}}\right],
$$

which separates impulsive internal waves into gravity waves of frequency $N|z| / r$ and buoyancy oscillations of frequency $N$.

\subsection{Non-oscillating source}

Consider first a non-oscillating source $\left(\omega_{0}=0\right)$, and assume, with implications to be discussed later, that for almost all epochs $\tau$ in (3.6) it is legitimate to set $N(t-\tau) \gg 1$. 
Then, replacing the Green's function by its asymptotic expansion yields

$$
\psi(\boldsymbol{r}, t) \sim-\frac{1}{(2 \pi)^{\frac{3}{2}} N} \int_{0}^{t} \frac{m_{0}(\tau)}{R_{h}(\tau)}\left\{\frac{\cos \left[N(t-\tau) \frac{|Z(\tau)|}{R(\tau)}-\frac{\pi}{4}\right]}{\left[N(t-\tau) \frac{|Z(\tau)|}{R(\tau)}\right]^{\frac{1}{2}}}+\frac{\sin \left[N(t-\tau)-\frac{\pi}{4}\right]}{[N(t-\tau)]^{\frac{1}{2}}}\right\} \mathrm{d} \tau
$$

where $\boldsymbol{R}(\tau)=\boldsymbol{r}-\boldsymbol{r}_{0}(\tau)$ denotes the position at $\tau$ of the observation point relative to the source (see figure 1). The wavy nature of the internal potential is now explicit, as is the separation of gravity waves - identical to those found by Sturova (1980) and Chashechkin \& Makarov (1984) -- and buoyancy oscillations.

Now, because of the assumption $N t \gg 1$ implicit in $N(t-\tau) \gg 1$, the integral (3.8) can be further evaluated by the method of stationary phase (Bleistein 1984, \$2.7). Three contributions arise, only two of which are meaningful by virtue of $N(t-\tau) \gg 1$, which prevents $\tau$ from approaching the endpoint $t$. Associated with the endpoint 0 are transient waves generated by the impulsive start, and given by

$$
\begin{aligned}
\psi_{t}(\boldsymbol{r}, t) \sim & -\frac{m_{0}(0)}{(2 \pi)^{\frac{3}{2}} N^{2} r_{h}} \frac{\frac{r}{|z|}}{1-\frac{\boldsymbol{v}_{0}(0) t}{r} \cdot\left(\frac{\boldsymbol{r}}{r}-\frac{r}{z} \boldsymbol{e}_{z}\right)} \frac{\sin \left(N t \frac{|z|}{r}-\frac{\pi}{4}\right)}{\left(N t \frac{|z|}{r}\right)^{\frac{1}{2}}} \\
& +\frac{m_{0}(0)}{(2 \pi)^{\frac{3}{2}} N^{2} r_{h}} \frac{\cos \left(N t-\frac{\pi}{4}\right)}{(N t)^{\frac{1}{2}}},
\end{aligned}
$$

where $v_{0}(\tau)=\mathrm{d} r_{0} / \mathrm{d} \tau$ is the velocity of the source and $e_{z}$ is a unit vector along the $z$-axis. As remarked by Bretherton $(1967$, p. 557$)$, the gravity waves and buoyancy oscillations composing such transients differ only by a phase shift and an amplitude factor from the gravity waves and buoyancy oscillations appearing in the Green's function. Accordingly, for the corresponding pressure and velocity, buoyancy oscillations turn out to be negligible compared with gravity waves, at least in the early stages of motion when the point source model makes sense (I, $\$ 7.2$ and 8.3).

The third, most significant, contribution represents permanent waves continuously built up by the motion of the source. It corresponds to the point $\tau_{s}$ where the phase

$$
\Phi(\tau ; \boldsymbol{r}, t)=N(t-\tau) \frac{|Z(\tau)|}{R(\tau)}
$$

of gravity waves is stationary. From the condition of stationarity $\partial \Phi /\left.\partial \tau\right|_{\tau=\tau_{s}}=0$, written as the implicit equation

$$
t-\tau_{s}=\frac{R\left(\tau_{s}\right)}{\boldsymbol{v}_{0}\left(\tau_{s}\right) \cdot\left[\frac{\boldsymbol{R}\left(\tau_{s}\right)}{\boldsymbol{R}\left(\tau_{s}\right)}-\frac{R\left(\tau_{s}\right)}{Z\left(\tau_{s}\right)} \boldsymbol{e}_{z}\right]},
$$

of the form (1.2), we identify $\tau_{s}$ as the retarded time and the associated internal potential, namely

$$
\psi_{p}(\boldsymbol{r}, t) \sim-\frac{m_{0}\left(\tau_{s}\right)}{2 \pi N^{2}} \frac{R\left(\tau_{s}\right)}{R_{h}\left(\tau_{s}\right)\left|Z\left(\tau_{s}\right)\right|} \frac{\cos \left[\frac{N}{c_{g}}\left|Z\left(\tau_{s}\right)\right|-\frac{\pi}{2} H(-A)\right]}{|A|^{\frac{1}{2}}},
$$


with

$$
A=R \frac{\gamma_{0}}{c_{g}^{2}} \cdot\left(\frac{\boldsymbol{R}}{R}-\frac{R}{Z} \boldsymbol{e}_{z}\right)-\left(\frac{\boldsymbol{v}_{0}}{c_{g}} \times \frac{\boldsymbol{R}}{R}\right)^{2}+\left.2 \frac{\boldsymbol{v}_{0}}{c_{g}} \cdot \frac{R}{Z} \boldsymbol{e}_{z}\right|_{\tau=\tau_{s}},
$$

as a Liénard-Wiechert potential for internal waves, where $\gamma_{0}(\tau)=\mathrm{d}^{2} \boldsymbol{r}_{0} / \mathrm{d} \tau^{2}$ is the acceleration of the source and

$$
\boldsymbol{c}_{\mathrm{g}}=\frac{\boldsymbol{R}\left(\tau_{s}\right)}{t-\tau_{s}}=\boldsymbol{v}_{0}\left(\tau_{s}\right) \cdot\left[\frac{\boldsymbol{R}\left(\tau_{s}\right)}{R\left(\tau_{s}\right)}-\frac{\boldsymbol{R}\left(\tau_{s}\right)}{Z\left(\tau_{s}\right)} \boldsymbol{e}_{z}\right] \frac{\boldsymbol{R}\left(\tau_{s}\right)}{R\left(\tau_{s}\right)}
$$

is the group velocity.

Of all the impulses generated by the source at the various points of its path, the principle of stationary phase has filtered out the only impulse, generated at the retarded time $\tau_{s}$, which can reach the point $\boldsymbol{r}$ at time $t$. Thus, permanent waves have the same structure as gravity waves due to a point impulsive source, investigated in I, except for the replacement of $\boldsymbol{r}$ and $t$ by $\boldsymbol{R}\left(\tau_{s}\right)$ and $t-\tau_{s}$. This is in particular true of the frequency $\omega$ and wavenumber vector $\boldsymbol{k}$, deduced from (3.10) as

$$
\begin{gathered}
\omega=\left.\frac{\partial \Phi}{\partial t}\right|_{\tau=\tau_{s}}=N \frac{|Z|}{R}, \\
\boldsymbol{k}=-\left.\nabla \Phi\right|_{\tau=\tau_{s}}=\frac{N}{c_{g}} \frac{\boldsymbol{R}}{R} \times\left(\frac{\boldsymbol{R}}{R} \times \boldsymbol{e}_{z}\right) \operatorname{sgn} Z=\frac{\omega}{c_{g}}\left(\frac{\boldsymbol{R}}{R}-\frac{R}{Z} \boldsymbol{e}_{z}\right),
\end{gathered}
$$

and of the wavelength $\quad \lambda=\frac{2 \pi}{k}=2 \pi \frac{c_{g}}{N} \frac{R}{R_{h}}$.

Here the subscript $p$ has been omitted, and all the quantities involving a time $\tau$ are taken implicitly at time $\tau_{s}$. Moreover, as a consequence of the motion of the source, a new relation can be written between $\omega$ and $\boldsymbol{k}$, namely the Doppler relation

$$
\omega=\boldsymbol{v}_{0} \cdot \boldsymbol{k}
$$

and the phase $N|Z| / c_{g}$, evaluated at $\tau_{s}$, which appears in (3.12), is just the phase $\omega\left(t-\tau_{s}\right)$ obtained by Stevenson (1973), and for constant velocity $\boldsymbol{v}_{0}$ of the source it reduces to the phase $-\boldsymbol{k} \cdot\left(\boldsymbol{r}-\boldsymbol{v}_{0} t\right)$ obtained by Lighthill $(1967,1978, \S 4.12)$.

The corresponding pressure and velocity follow from the differentiation of the internal potential according to (3.2), taking into account that the only significant asymptotic terms arise from the variations of the phase, governed by (3.15)-(3.16); they can, alternatively, be deduced from the pressure and velocity I (7.7)-(7.8) associated with the Green's function. Both methods yield

$$
\begin{gathered}
P(r, t) \sim \frac{\rho_{0} N m_{0}}{2 \pi} \frac{R_{h}}{R^{2}} \frac{\cos \left[\frac{N}{c_{g}}|Z|+\frac{\pi}{2} H(A)\right]}{|A|^{\frac{1}{2}}}, \\
\boldsymbol{v}(\boldsymbol{r}, t) \sim \frac{N m_{0}}{2 \pi c_{g}} \frac{R_{h}}{R^{2}} \frac{\boldsymbol{R}}{R} \frac{\cos \left[\frac{N}{c_{g}}|Z|+\frac{\pi}{2} H(A)\right]}{|A|^{\frac{1}{2}}} .
\end{gathered}
$$

As for the point impulsive source, the pressure and velocity oscillate in phase and verify

$$
v \sim \frac{P}{\rho_{0} c_{g}} \frac{R}{R}
$$


Permanent waves take up the whole region $0<\tau_{s}<t$ where the retarded time exists and makes sense. Transient waves, negligible there, are observed outside. On the boundary $\tau_{s}=0$ both wave systems merge, in such a way that buoyancy oscillations do not change while gravity waves combine into one half of the permanent waves (3.12). In the framework of the method of stationary phase this corresponds to the coalescence of the point of stationary phase with an endpoint. A uniform expansion describing the vicinity of the surface $\tau_{s}=0$ would then involve error functions (Bleistein 1966); this is just the result obtained by Chashechkin \& Makarov (1984) in a particular case.

So far, two hypotheses have been used. The first of them, $N(t-\tau) \gg 1$, appears now as the 'far-field' condition

$$
\frac{N}{c_{g}} R \gg 1
$$

or, by (3.17), omitting the angular factor $R_{h} / R$, as

$$
R \gg \lambda,
$$

which states that waves are observed at many wavelengths from the source path. The second hypothesis, which also follows from $N(t-\tau) \gg 1$, is simply the large-time condition

$$
N t \gg 1,
$$

which states that waves are observed many periods after the motion has begun. Since the stationary phase description of a wave field is generally valid even for moderate values of the assumed large parameter, we can expect (3.22) to hold whenever $N R / c_{\mathrm{g}} \gtrsim 1$. It is difficult to find out a priori (i.e. without studying a particular motion) where this is the case, since the variations of $R$ and $c_{\mathrm{g}}$ are not independent, and since these quantities are precisely determined by the method the validity of which they are supposed to be assessing. We may, however, consider two regions where (3.22) surely breaks down, namely $R \rightarrow 0$ and $c_{\mathrm{g}} \rightarrow \infty$, and examine how they are approached as the observer leaves the far field; during this transition it is legitimate to set $t-\tau_{s}$ fixed and non-zero. In both cases, assumptions more basic than (3.22) turn out to be violated; since $R \rightarrow 0$, which corresponds to the vicinity of the source path, implies $\lambda \rightarrow 0$ and invalidates the point source model, while $c_{\mathrm{g}} \rightarrow \infty$ implies $\lambda \rightarrow \infty$ and invalidates the Boussinesq approximation.

\subsection{Oscillating source}

When the source simultaneously moves $\left(v_{0} \neq 0\right)$ and oscillates $\left(\omega_{0} \neq 0\right)$, the factor $\mathrm{e}^{\mathrm{i} \omega_{0} \tau}$ arising in the source strength modifies the definition of the phases of gravity waves and buoyancy oscillations. Integral (3.8) becomes

$$
\begin{aligned}
& \psi(\boldsymbol{r}, t) \sim-\frac{1}{2^{\frac{5}{2}} \pi^{\frac{3}{2}} N} \sum \int_{0}^{t} \mathrm{~d} \tau \frac{m_{0}(\tau)}{R_{h}(\tau)} \\
& \times\left\{\frac{\exp \left\{\mathrm{i}\left[\omega_{0} \tau \pm N(t-\tau) \frac{|Z(\tau)|}{R(\tau)} \mp \frac{\pi}{4}\right]\right\}}{\left[N(t-\tau) \frac{|Z(\tau)|}{R(\tau)}\right]^{\frac{1}{2}}} \mp \mathrm{i} \frac{\exp \left\{\mathrm{i}\left[\omega_{0} \tau \pm N(t-\tau) \mp \frac{\pi}{4}\right]\right\}}{[N(t-\tau)]^{\frac{1}{2}}}\right\},
\end{aligned}
$$


where $\sum_{ \pm}$denotes summation over the signs + and - . Gravity waves are split in two systems of waves, with phases

$$
\Phi_{ \pm}(\tau ; \boldsymbol{r}, t)=\omega_{0} \tau \pm N(t-\tau) \frac{|Z(\tau)|}{R(\tau)},
$$

so that permanent waves are also split in two systems of waves, each of which with its own retarded time $\tau_{ \pm}$satisfying

$$
t-\tau_{ \pm}=\frac{\left[1 \mp \frac{\omega_{0}}{N} \frac{R\left(\tau_{ \pm}\right)}{\left|Z\left(\tau_{ \pm}\right)\right|}\right] R\left(\tau_{ \pm}\right)}{\boldsymbol{v}_{0}\left(\tau_{ \pm}\right) \cdot\left[\frac{\boldsymbol{R}\left(\tau_{ \pm}\right)}{R\left(\tau_{ \pm}\right)}-\frac{R\left(\tau_{ \pm}\right)}{Z\left(\tau_{ \pm}\right)} \boldsymbol{e}_{z}\right]}
$$

its own group velocity

$$
\boldsymbol{c}_{ \pm}=\frac{\boldsymbol{v}_{0}\left(\tau_{ \pm}\right) \cdot\left[\frac{\boldsymbol{R}\left(\tau_{ \pm}\right)}{R\left(\tau_{ \pm}\right)}-\frac{R\left(\tau_{ \pm}\right)}{Z\left(\tau_{ \pm}\right)} e_{z}\right]}{1 \mp \frac{\omega_{0}}{N} \frac{R\left(\tau_{ \pm}\right)}{\left|Z\left(\tau_{ \pm}\right)\right|}} \frac{\boldsymbol{R}\left(\tau_{ \pm}\right)}{R\left(\tau_{ \pm}\right)}
$$

and its own internal potential

$$
\psi_{ \pm}(\boldsymbol{r}, t) \sim-\frac{m_{0}\left(\tau_{ \pm}\right)}{4 \pi N^{2}} \frac{R\left(\tau_{ \pm}\right)}{R_{h}\left(\tau_{ \pm}\right)\left|Z\left(\tau_{ \pm}\right)\right|} \frac{\exp \left\{\mathrm{i}\left[\omega_{0} \tau_{ \pm} \pm \frac{N}{c_{ \pm}}\left|Z\left(\tau_{ \pm}\right)\right| \mp \frac{\pi}{2} H\left(-A_{ \pm}\right)\right]\right\}}{\left|A_{ \pm}\right|^{\frac{1}{2}}},
$$

where

$$
\begin{aligned}
A_{ \pm}=R \frac{\gamma_{0}}{c_{ \pm}^{2}} \cdot\left(\frac{\boldsymbol{R}}{R}-\frac{R}{Z} \boldsymbol{e}_{z}\right)- & \left(\frac{\boldsymbol{v}_{0}}{c_{ \pm}} \times \frac{\boldsymbol{R}}{R}\right)^{2} \\
& +\left.2\left(1 \mp \frac{\omega_{0}}{N} \frac{R}{|Z|}\right)\left(\frac{\boldsymbol{v}_{0}}{c_{ \pm}} \cdot \frac{R}{Z} \boldsymbol{e}_{z} \mp \frac{\omega_{0}}{N} \frac{R}{|Z|}\right)\right|_{\tau=\tau_{ \pm}}
\end{aligned}
$$

We interpret this as a beating between the waves generated by the motion of the source alone and those generated by the oscillations of the source alone. Sum and difference waves are created, with sum and difference frequencies

$$
\omega_{ \pm}= \pm N \frac{\left|Z_{ \pm}\right|}{R_{ \pm}}
$$

respectively positive and negative, sum and difference wavenumber vectors

$$
\boldsymbol{k}_{ \pm}= \pm \frac{N}{c_{ \pm}} \frac{\boldsymbol{R}_{ \pm}}{\boldsymbol{R}_{ \pm}} \times\left(\frac{\boldsymbol{R}_{ \pm}}{\boldsymbol{R}_{ \pm}} \times \boldsymbol{e}_{z}\right) \operatorname{sgn} Z_{ \pm}=\frac{\omega_{ \pm}}{c_{ \pm}}\left(\frac{\boldsymbol{R}_{ \pm}}{\boldsymbol{R}_{ \pm}}-\frac{\boldsymbol{R}_{ \pm}}{Z_{ \pm}} \boldsymbol{e}_{z}\right),
$$

and sum and difference wavelengths

$$
\lambda_{ \pm}=2 \pi \frac{c_{ \pm}}{N} \frac{R_{ \pm}}{R_{\mathrm{h}_{ \pm}}}
$$

where the subscript \pm implies evaluation of the concerned quantity at time $\tau_{ \pm}$. Both wave systems satisfy the Doppler relation

$$
\omega_{ \pm}=\omega_{0}+\boldsymbol{v}_{0_{ \pm}} \cdot \boldsymbol{k}_{ \pm}
$$

and their phases $\omega_{0} \tau_{ \pm} \pm N\left|Z_{ \pm}\right| / c_{ \pm}$coincide as well with those obtained by Stevenson 
(1973), $\omega_{0} \tau_{ \pm}+\omega_{ \pm}\left(t-\tau_{ \pm}\right)$, and, for constant source velocity $\boldsymbol{v}_{0}$, with those obtained by Lighthill $(1967,1978, \S 4.12), \omega_{0} t-\boldsymbol{k}_{ \pm} \cdot\left(\boldsymbol{r}-\boldsymbol{v}_{0} t\right)$.

The pressure and velocity are

$$
\begin{aligned}
& P_{ \pm}(\boldsymbol{r}, t) \sim \frac{\rho_{0} N m_{0_{ \pm}}}{4 \pi} \frac{R_{\mathrm{h}_{ \pm}}}{R_{ \pm}^{2}} \frac{\exp \left\{\mathrm{i}\left[\omega_{0} \tau_{ \pm} \pm \frac{N}{c_{ \pm}}\left|Z_{ \pm}\right| \pm \frac{\pi}{2} H\left(A_{ \pm}\right)\right]\right\}}{\left|A_{ \pm}\right|^{\frac{1}{2}}} \\
& \boldsymbol{v}_{ \pm}(\boldsymbol{r}, t) \sim \frac{N m_{0_{ \pm}}}{4 \pi c_{ \pm}} \frac{R_{\mathrm{h}_{ \pm}}}{R_{ \pm}^{2}} \frac{\boldsymbol{R}_{ \pm}}{R_{ \pm}} \frac{\exp \left\{\mathrm{i}\left[\omega_{0} \tau_{ \pm} \pm \frac{N}{c_{ \pm}}\left|Z_{ \pm}\right| \pm \frac{\pi}{2} H\left(A_{ \pm}\right)\right]\right\}}{\left|A_{ \pm}\right|^{\frac{1}{2}}} .
\end{aligned}
$$

For each wave system, they oscillate in phase and verify

$$
\boldsymbol{v}_{ \pm} \sim \frac{\boldsymbol{P}_{ \pm}}{\rho_{0} \mathcal{c}_{ \pm}} \frac{\boldsymbol{R}_{ \pm}}{R_{ \pm}} .
$$

For a non-oscillating source $\left(\omega_{0}=0\right)$ both sum and difference waves are present, with identical retarded times $\tau_{ \pm}$, opposite frequencies $\omega_{ \pm}$and wavenumber vectors $\boldsymbol{k}_{ \pm}$, and complex conjugate contributions $\psi_{ \pm}, P_{ \pm}$and $\boldsymbol{v}_{ \pm}$to the wave field. Accordingly the two wave systems combine into one, the sign of whose phase is arbitrary, as reflected by the cosines replacing in (3.12) and (3.19)-(3.20) the complex exponentials of (3.28) and (3.34)-(3.35). In $\S 3.1$ the determination (3.10) of $\Phi$ has been chosen to yield a positive $\omega$, and thus a description of internal waves in terms only of sum waves has been adopted (see Lighthill 1978, p. 353). For a non-moving source $\left(v_{0}=0\right)$ difference waves are absent, as a consequence of the representation $\mathrm{e}^{\mathrm{i} \omega_{0} \tau}$ of the oscillations, and the retarded time equation (3.26) reduces to the equation $\omega_{0}=N|z| / r$ defining the characteristic cone.

\section{Internal waves generated by a uniformly moving source}

When the source is purely oscillatory $\left(m_{0}=\right.$ const) and moves uniformly $\left(v_{0}=\right.$ const), a major simplification arises: the permanent wave field is stationary, in that it moves and oscillates with the source. This follows from introducing the position $\boldsymbol{r}_{1}=\boldsymbol{r}-\boldsymbol{v}_{0} t$ relative to the source at $t$, and writing $\boldsymbol{R}(\tau)=\boldsymbol{r}_{1}+\boldsymbol{v}_{0}(t-\tau)$. Then, by (3.26), $t-\tau_{ \pm}$and $\boldsymbol{R}\left(\tau_{ \pm}\right)$depend only on $\boldsymbol{r}_{1}$, so that the internal potential (3.28), pressure (3.34) and velocity (3.35) depend only on $\boldsymbol{r}_{1}$ and, through the factor $\mathrm{e}^{\mathrm{i} \omega_{0} t}$, on $t$.

Most studies of moving sources of internal waves consider uniform motion, and use the stationarity of the permanent wave field to attack its calculation on a steady-state basis. This amounts to applying the limit $t \rightarrow \infty$ with $\boldsymbol{r}_{1}$ fixed to (3.6), which becomes

$$
\psi(\boldsymbol{r}, t)=m_{0} \mathrm{e}^{\mathrm{i} \omega_{0} t} \int \mathrm{e}^{-\mathrm{i} \omega_{0} \tau} G\left(\boldsymbol{r}_{1}+\boldsymbol{v}_{0} \tau, \tau\right) \mathrm{d} \tau .
$$

In the present section, following Gorodtsov \& Teodorovich (1980), we briefiy investigate the consequences of (4.1). Our starting point is the set of formulae given in I for the spatial and temporal Fourier transforms of the Green's function, defined by

$$
G(\boldsymbol{r}, t)=\frac{1}{(2 \pi)^{4}} f \mathrm{~d} \omega \int \mathrm{d}^{3} k G(\boldsymbol{k}, \omega) \mathrm{e}^{\mathrm{j}(\omega t-\boldsymbol{k} \cdot \boldsymbol{r})},
$$

where the sign - indicates indentation of the integration path below the real singularities of the integrand, in compliance with causality which requires that $G(r, \omega)$ be 
analytic in the lower half of the complex $\omega$-plane (see I, $\S 3.2$, and Crighton \& Oswell 1991).

From the substitution of (4.2) in (4.1), and use of I (5.6)-(5.7), we have

$$
\begin{aligned}
\psi(\boldsymbol{r}, t) & =\frac{m_{0}}{(2 \pi)^{3}} \mathrm{e}^{\mathrm{i} \omega_{0} t} \lim _{\epsilon \rightarrow 0_{+}} \int G\left(\boldsymbol{k}, \omega_{0}+\boldsymbol{v}_{0} \cdot \boldsymbol{k}-\mathrm{i} \epsilon\right) \mathrm{e}^{-\mathrm{i} \boldsymbol{k} \cdot \boldsymbol{r}_{1}} \mathrm{~d}^{3} k, \\
& =\frac{m_{0}}{(2 \pi)^{3}} \mathrm{e}^{\mathrm{i} \omega_{0} t} \lim _{\epsilon \rightarrow 0_{+}} \int \frac{\mathrm{e}^{-\mathrm{i} \boldsymbol{k} \cdot \boldsymbol{r}_{1}}}{\left(\omega_{0}+\boldsymbol{v}_{0} \cdot \boldsymbol{k}-\mathrm{i} \epsilon\right)^{2} k^{2}-N^{2} k_{h}^{2}} \mathrm{~d}^{3} k .
\end{aligned}
$$

This is just, applied to internal waves, the basic formula of Lighthill's $(1967,1978$, $\S 4.12)$ theory, and the addition of the infinitesimal negative imaginary part $-\epsilon$ to the frequency is just Lighthill's expression of the radiation condition.

We introduce now a system of coordinates $\left(x_{\|}, y_{1}, z_{\perp}\right)$, with the $x_{\|}$-axis parallel to the path of the source and the $\left(y_{\perp}, z_{\perp}\right)$-plane perpendicular to it, and write $\boldsymbol{r}_{\perp}=\left[0, y_{\perp}, z_{\perp}\right]$. Then, integration of $(4.3 a)$ in the transverse wavenumber plane yields

$$
\psi(\boldsymbol{r}, t)=\frac{m_{0}}{2 \pi} \mathrm{e}^{\mathrm{i} \omega_{0} t} f G\left(k_{\|}, y_{\perp}, z_{\perp}, \omega_{0}+\bar{v}_{0} k_{\Downarrow}\right) \mathrm{e}^{-i k_{\|} x_{\Downarrow}} \mathrm{d} k_{\|} \quad\left(\bar{v}_{0} \lessgtr 0\right),
$$

where $\bar{v}_{0}$ is the algebraic value of the source velocity projected onto the $x_{\|}$-axis, and the sign $-(\neg)$ indicates deformation of the integration path below (above) the real singularities of the integrand for positive (negative) $\bar{v}_{0}$, respectively. For a steady source $\left(\omega_{0}=0\right)$, the symmetry properties of the Green's function $G(\boldsymbol{k}, \omega)=$ $G(-\boldsymbol{k}, \omega)=G^{\star}(\boldsymbol{k},-\omega)$, where ${ }^{\star}$ denotes a complex conjugate, imply that, moreover,

$$
\psi(\boldsymbol{r}, t)=\frac{m_{0}}{\pi} \operatorname{Re} f_{0}^{\infty} G\left(k_{\|}, y_{\perp}, z_{\perp}, \bar{v}_{0} k_{\|}\right) \mathrm{e}^{-\mathrm{i} k_{\|} x_{\|}} \mathrm{d} k_{\|} \quad\left(\bar{v}_{0} \lessgtr 0\right),
$$

restricting the wavenumber range of (4.4) to $\operatorname{Re} k_{\|}>0$. When some particular motion is considered and the explicit form of $G\left(k_{\|}, y_{\perp}, z_{\perp}, \omega\right)$ is used, equations (4.4) and (4.5) reduce to just the basic formulae of the integral method, and the deformation of the integration path expresses just the radiation condition.

This deformation, in fact, concerns only the singularities associated with the indirect dependence of the integrand of (4.4) on $k_{\|}$, through the Doppler relation $\omega=\omega_{0}+\bar{v}_{0} k_{\|}$. If those were its only singularities throughout the complex $k_{\|}$-plane, closure of the integration contour in the half-plane $\operatorname{Im}\left(\bar{v}_{0} k_{\|}\right)<0$ would imply that $\psi(r, t)=0$ for $\bar{v}_{0} x_{\|}>0$, in other terms that no waves would be present upstream of the source. However, in the integrand of (4.4) there is also a second, direct, dependence on $k_{\|}$; namely, as revealed by $\mathrm{I}(5.8)$ and $\mathrm{I}(5.10), G\left(k_{\|}, y_{\perp}, z_{\perp}, \omega\right)$ is a function of the complex variable $k_{\|}$singular at the origin $k_{\|}=0$. This gives rise to upstream waves.

\section{Uniform horizontal motion of a steady source}

The next three sections apply both the unsteady theory of $\S 3$ and the steady theory of $\S 4$ to three problems of increasing analytical complexity. All calculations are presented in summarized form; further detail may be found in Voisin (1991a).

First we consider the uniform horizontal motion of a steady source, with applications as well to the generation of lee waves and to the wave resistance of ships (Miles $1969 \mathrm{c})$. By steady source we mean a source of constant strength $\left(m_{0}=\right.$ const and $\left.\omega_{0}=0\right)$, and by uniform horizontal motion we mean motion at the velocity $\boldsymbol{v}_{0}=-U \boldsymbol{e}_{x}$, with $U$ constant and positive. A moving system of coordinates $\left(x_{1}, y, z\right)$ is used, with the $x_{1}$-axis horizontal and opposite to the motion, and the $z$-axis directed 


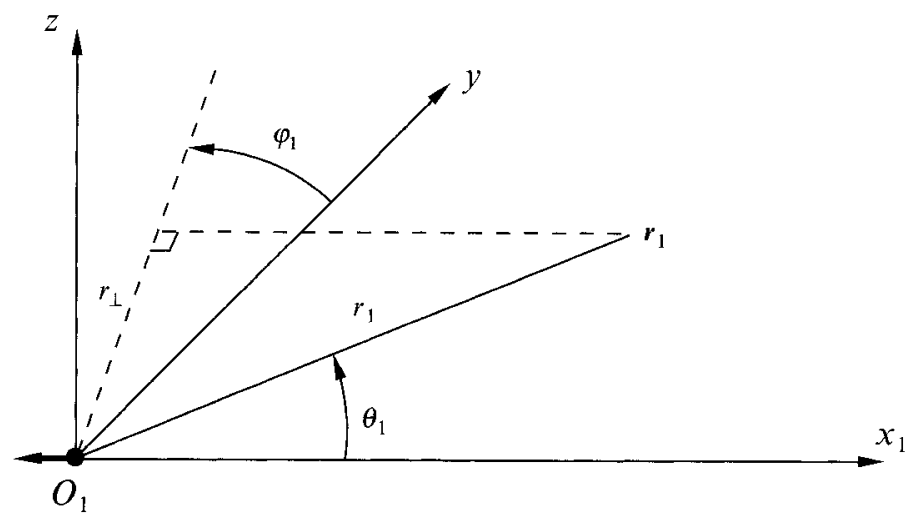

FIGURE 2. Coordinate system for uniform horizontal motion.

vertically upwards; $\boldsymbol{e}_{x}$ is a unit vector along the $x_{1}$-axis. We also introduce spherical coordinates $\left(r_{1}, \theta_{1}, \varphi_{1}\right)$ defined by

$$
\begin{gathered}
x_{1}=x+U t=r_{1} \cos \theta_{1}, \quad y=r_{1} \sin \theta_{1} \cos \varphi_{1}, \quad z=r_{1} \sin \theta_{1} \sin \varphi_{1}, \\
r_{1}=\left(y^{2}+z^{2}\right)^{\frac{1}{2}}=r_{1} \sin \theta_{1},
\end{gathered}
$$

and represented in figure 2 .

\subsection{Unsteady approach}

The way that the wave field is built up follows from the expression of the retarded time, namely

$$
t-\tau_{s}=\frac{r_{1}^{2}}{U x_{1}}=\frac{r_{1}}{U \cos \theta_{1}},
$$

and from the causality condition $0<\tau_{s}<t$. First, the distance travelled by permanent waves between their emission at $\tau_{s}$ and their reception at $t$ is, in Cartesian components,

$$
\boldsymbol{R}\left(\tau_{s}\right)=r_{1} \tan \theta_{1}\left[-\sin \theta_{1}, \cos \theta_{1} \cos \varphi_{1}, \cos \theta_{1} \sin \varphi_{1}\right],
$$

and is transverse, so that the current position $O_{1}$ of the source, its position $S$ at emission and the position $M$ of reception form a triangle right-angled at $M$ (figure 3), and all the waves emitted at point $S$ are situated at time $t$ on the sphere of diameter $O_{1} S$. Secondly, the waves generated since the beginning of the motion at point $O$ are found downstream, inside the sphere of diameter $O_{1} O$. Out of the sphere, transient waves generated by the impulsive start are observed; on the sphere the two systems of waves merge (figure 4), consistently with the experiments of Stevenson \& Thomas (1969), Stevenson (1973) and Peat \& Stevenson (1975), and the calculations of Chashechkin \& Makarov (1984).

Once $\boldsymbol{R}\left(\tau_{s}\right)$ is known, the determination of the waves is straightforward but is essentially a rederivation of existing results. We just mention here, accordingly, the most significant of them. These include the frequency and wavelength

$$
\omega=N \cos \theta_{1}\left|\sin \varphi_{1}\right|, \quad \lambda=2 \pi \frac{U}{N} \frac{\sin \theta_{1}}{\left(\sin ^{2} \theta_{1}+\cos ^{2} \theta_{1} \cos ^{2} \varphi_{1}\right)^{\frac{1}{2}}},
$$

bounded and smaller than $N$ and $2 \pi U / N$, respectively; the group velocities in the fixed and moving reference frames

$$
\boldsymbol{c}_{g}=U \sin \theta_{1}\left[-\sin \theta_{1}, \cos \theta_{1} \cos \varphi_{1}, \cos \theta_{1} \sin \varphi_{1}\right],
$$




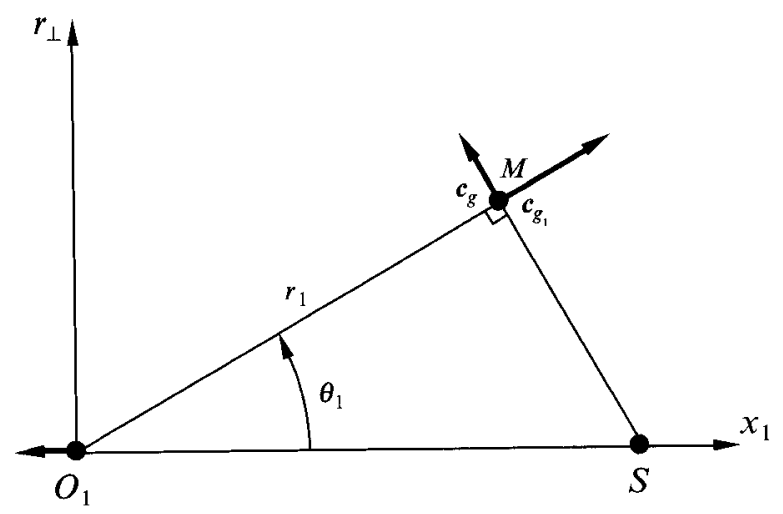

FIGURE 3. Geometry for internal wave radiation by a steady source in uniform horizontal motion, in a plane $\varphi_{1}=$ const.

$$
\boldsymbol{c}_{g_{1}}=\boldsymbol{c}_{g}+U \boldsymbol{e}_{x}=U \cos \theta_{1}\left[\cos \theta_{1}, \sin \theta_{1} \cos \varphi_{1}, \sin \theta_{1} \sin \varphi_{1}\right],
$$

respectively transverse and radial and both smaller than $U$; and the far-field velocity, for $N r_{1} / U \gg 1$,

$$
\boldsymbol{v}(\boldsymbol{r}, t) \sim H\left(x_{1}\right) \frac{N m_{0}}{2 \pi U r_{1}} \frac{\left(\sin ^{2} \theta_{1}+\cos ^{2} \theta_{1} \cos ^{2} \varphi_{1}\right)^{\frac{1}{2}}}{\sin \theta_{1}} \frac{\boldsymbol{R}}{R} \cos \left(\frac{N}{U} r_{1}\left|\sin \varphi_{1}\right|\right),
$$

where the steady-state limit $t \rightarrow \infty$ has been introduced, so that the sphere $r_{1}=$ $U t \cos \theta_{1}$ within which permanent waves are confined expands to the plane $x_{1}=0$ and is accounted for by the Heaviside function $H\left(x_{1}\right)$. As $x_{1} \rightarrow 0$ transient waves and permanent waves coalesce, expansion (5.7) being then non-uniform; for $x_{1}=0 \mathrm{a}$ factor $\frac{1}{2}$ must be incorporated in its right-hand side.

Equivalent forms of the waves have been obtained by Miles (1971), Dokuchaev \& Dolina (1977), Sturova (1978), Makarov \& Chashechkin (1981), Janowitz (1984) and Umeki \& Kambe (1989). They differ slightly from the results of Wurtele (1957) and Wu (1965), whereas the only detailed study of the asymptotic wave field is precisely attributable to Wurtele. It is, thus, of interest here to repeat this study. Since the velocity vector, parallel to $R\left(\tau_{s}\right)$, is transverse, we focus our attention on the vertical displacement $\zeta$, related to the vertical velocity by $v_{z}=\partial \zeta / \partial t$. Moreover, we for the moment replace our convenient but unrealistic model of a moving source of waves as the monopole $m(\boldsymbol{r}, t)=m_{0} \delta\left(x_{1}\right) \delta(y) \delta(z)$ by the classical model of a moving rigid body as the dipole

$$
m(r, t)=m_{d} \frac{\partial}{\partial x} \delta\left(x_{1}\right) \delta(y) \delta(z),
$$

of moment $m_{d}=U\left(V+V_{\star}\right)$ with $V$ the volume of the body and $\rho_{0} V_{\star}$ its added mass (Miles 1971; Lighthill 1978, $\S 1.7,1986, \S 8.3$ ). In the steady state, $\partial / \partial t=U \partial / \partial x$ and the dipolar vertical displacement reduces to $m_{d} /\left(U m_{0}\right)$ times the monopolar vertical velocity. Then, from (5.7), in Cartesian coordinates,

$$
\zeta(\boldsymbol{r}, t) \sim H\left(x_{1}\right) \frac{N m_{d}}{2 \pi U^{2}} \frac{x_{1} z}{r_{\perp}^{3}} \frac{\left(x_{1}^{2} y^{2}+r_{\perp}^{4}\right)^{\frac{1}{2}}}{x_{1}^{2}+r_{\perp}^{2}} \cos \left[\frac{N}{U} \frac{|z|}{r_{\perp}}\left(x_{1}^{2}+r_{\perp}^{2}\right)^{\frac{1}{2}}\right] .
$$




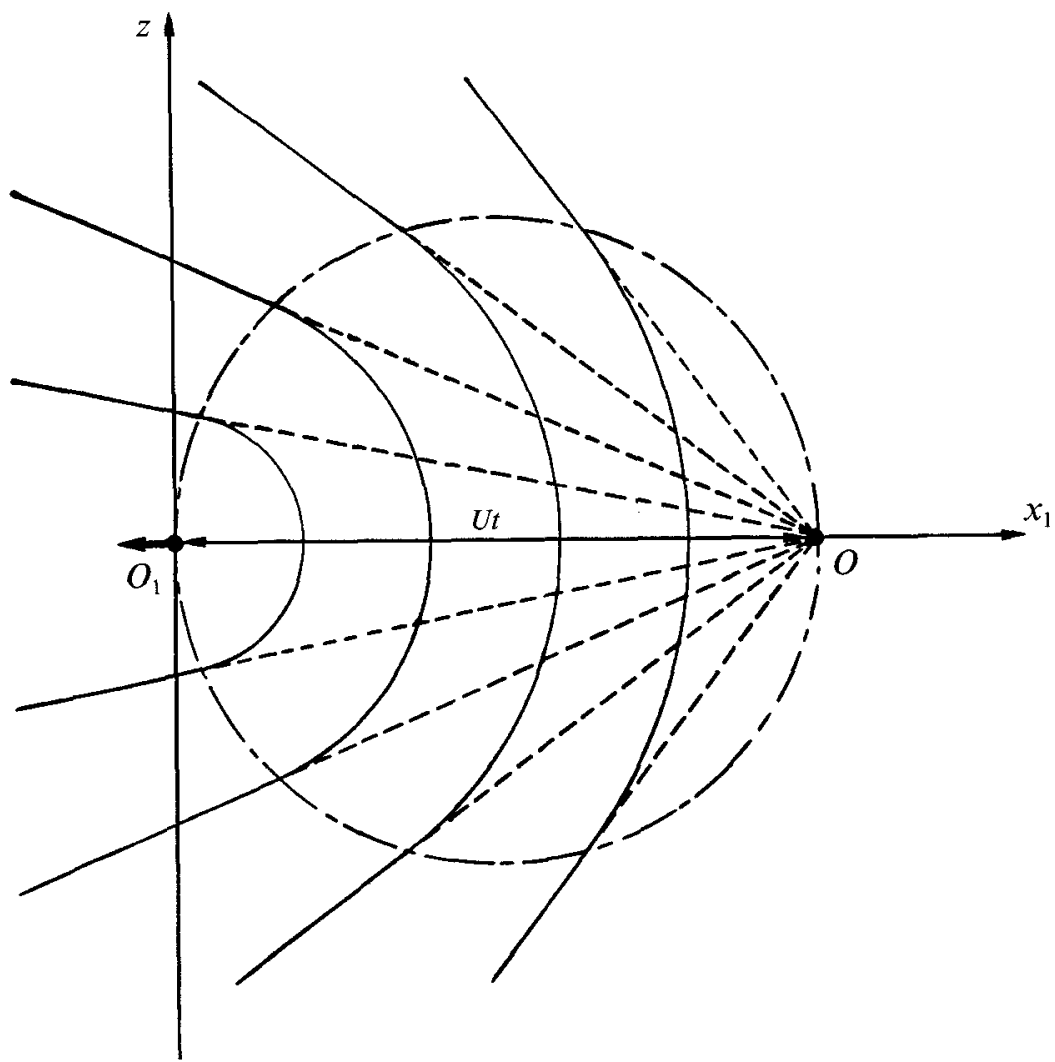

FIGURE 4. Wave systems generated by a steady source in uniform horizontal motion, in the plane $y=0$. Inside the sphere of diameter $O O_{1}$ joining the initial and present positions of the source, permanent waves are dominant and curves of constant phase are semi-circles $\Phi=N r_{1} / U$. Outside the sphere transient waves are observed, with radial curves of constant phase $\Phi=N t|z| / r$. On the sphere the two wave systems, shown for $N t / 2 \pi=5$ and $\Phi=2 n \pi$, merge.

The phase of the waves, namely

$$
\Phi=\frac{N}{U} r_{1}\left|\sin \varphi_{1}\right|=\frac{N}{U}|z|\left(\frac{x_{1}^{2}+y^{2}+z^{2}}{y^{2}+z^{2}}\right)^{\frac{1}{2}},
$$

is most easily visualized in planes of constant $x_{1}, y$ or $z$. In terms of the reduced coordinates $r_{\star}=\left(N r_{1}\right) /(U \Phi)$, surfaces of constant phase intersect horizontal planes $z_{\star}=$ const along the semi-hyperbolae

$$
\frac{x_{\star}^{2}}{1-z_{\star}^{2}}-\frac{y_{\star}^{2}}{z_{\star}^{2}}=1, \quad x_{\star}>0,
$$

and intersect the vertical plane $y_{\star}=0$ along the semi-circle

$$
x_{\star}^{2}+z_{\star}^{2}=1, \quad 0<x_{\star}<1,
$$

and the semi-line

$$
z_{\star}=0, \quad x_{\star}>1 .
$$

These intersections, represented in figure 5 and supplemented in figure 6 by a perspective view, are identical to those found theoretically by Wurtele (1957), Redekopp 


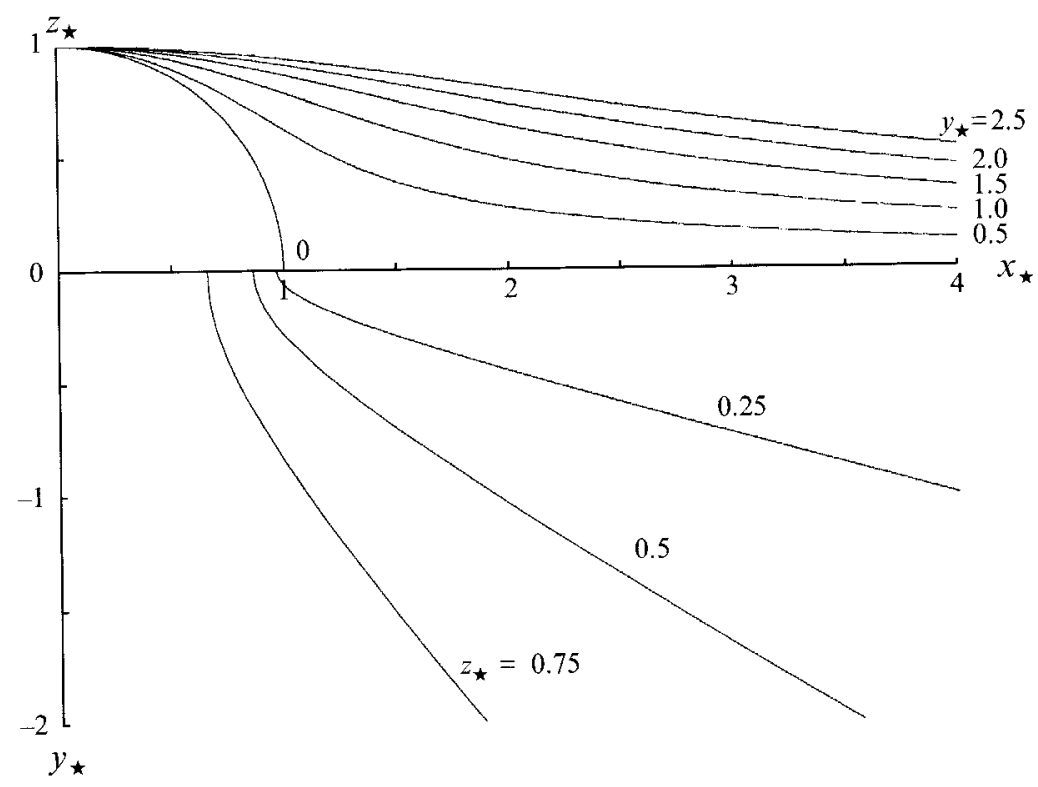

FIGURE 5. Curves of constant phase for uniform horizontal motion of a steady source. The curves, symmetric about the $x_{\star}$-axis, are drawn above and below it in planes $y_{\star}=$ const and $z_{\star}=$ const, respectively.

(1975), Rehm \& Radt (1975), Peat \& Stevenson (1975) and Thorpe (1975), and confirmed experimentally by Peat \& Stevenson (1975), Makarov \& Chashechkin (1981, 1982), Chashechkin (1989), Hopfinger et al. (1991) and Bonneton et al. (1993).

The structure of the displacement field combines these variations of the phase with the variations of the amplitude. In the plane $y=0$, it was represented by Wurtele (1957) as a set of isopycnals whose upstream tilt with $|z|$ reflects the circular wavecrests, in agreement with the visualizations of Castro et al. (1983), Castro (1987) and Hopfinger et al. (1991). In each plane $z=$ const, the amplitude is an extremum $\left(N m_{d}\right) /\left(4 \pi U^{2} z\right)$ at $\left(x_{1}=|z|, y=0\right)$, and at large distances downstream $\left(x_{1} \gg r_{\perp}\right)$ it becomes independent of $x_{1}$ and is a local extremum $\left(N m_{d}\right) /\left(3 \pi \sqrt{ } 3 U^{2} z\right)$ for $|y|=|z| / \sqrt{ } 2$ (Janowitz 1984). The resulting 'crescent'-shaped displacement field, reminiscent of the hyperbolic wavecrests, is shown in figure 7 for $N z / U=\frac{1}{2}, 1$, and coincides with laboratory (Hopfinger et al. 1991; Bonneton et al. 1993) as well as field (Wurtele 1957; Umeki \& Kambe 1989) observations.

Comparison with the numerical calculations of Sturova (1974) and Umeki \& Kambe (1989) reveals the domain of validity of those asymptotic results, say, $\left(x_{\star}>2, y_{*}>\right.$ $0.5)$, similar criteria $x_{\star}>2$ and $x_{\star}>4 \pi$ having been proposed by Wurtele (1957) and Sturova (1978), respectively. This confirms the discussion of $\S 3.1$, according to which the theory is valid sufficiently far from the source path. On this path, as pointed out by Miles (1971), expansions (5.7) and (5.9) diverge, as a consequence of the failure of both the asymptotic procedure (since $R \rightarrow 0$ ) and the point source model (since $\lambda \rightarrow 0$ ). In the intermediate zone $N x_{1} / U \gg N r_{\perp} / U \gg 1$ or $N r_{1} / U \gg 1 / \theta_{1} \gg 1$, that is near the source path but still in the far field, expansion (5.7) becomes

$$
\boldsymbol{v}(\boldsymbol{r}, t) \sim H\left(x_{1}\right) \frac{N m_{0}}{2 \pi U} \frac{|y|}{r_{\perp}^{2}} \frac{\boldsymbol{r}_{\perp}}{r_{\perp}} \cos \left[\frac{N}{U}|z|\left(\frac{x_{1}}{r_{\perp}}+\frac{r_{\perp}}{2 x_{1}}\right)\right] .
$$




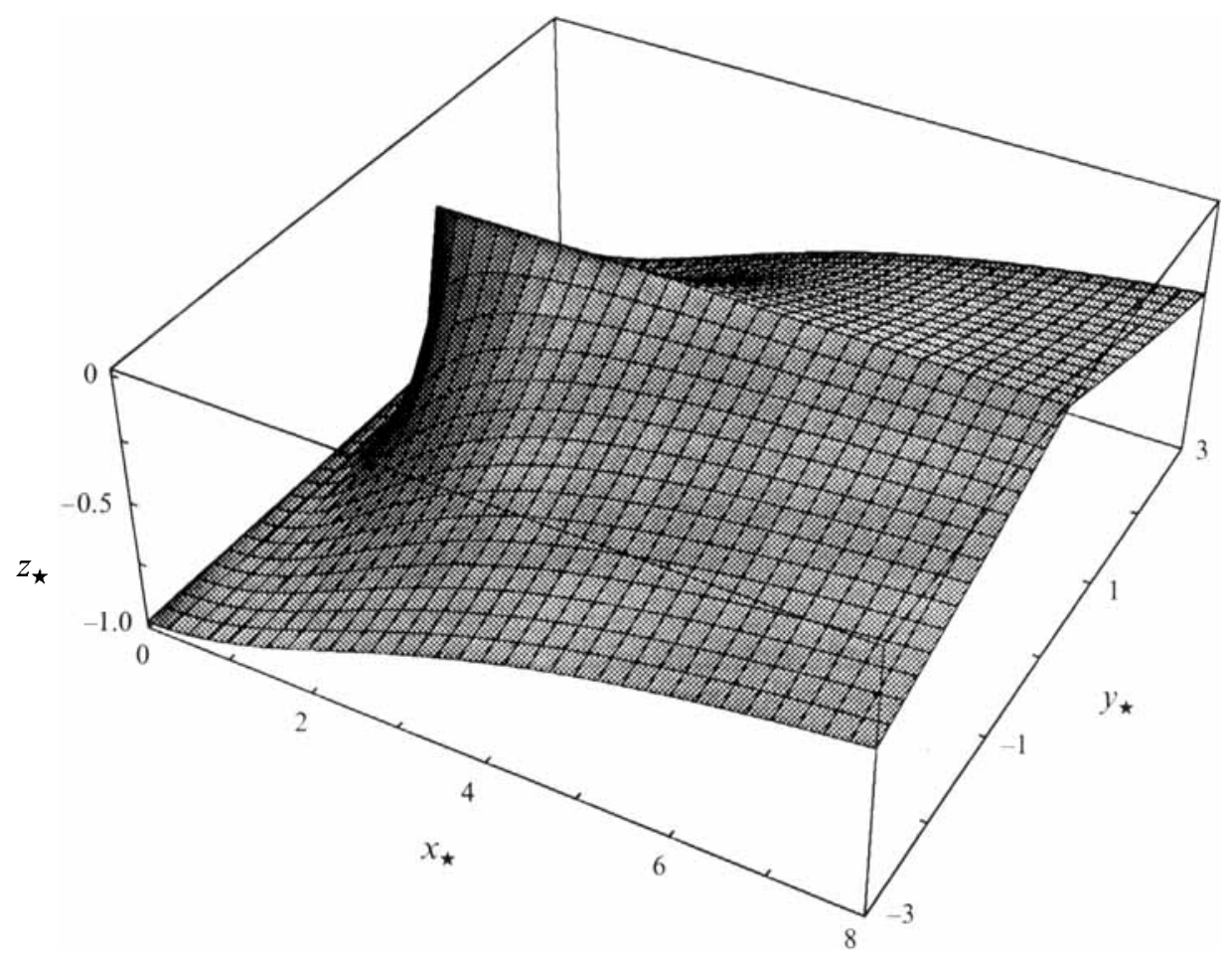

FIGURE 6. Surfaces of constant phase for uniform horizontal motion of a steady source. Only the lower half of these surfaces, symmetric about the plane $z_{\star}=0$, is shown.

To leading order, curves of constant phase simplify to semi-hyperbolae $x_{\star}^{2}-\left(y_{\star} / z_{\star}\right)^{2}=$ 1 in planes of constant $z_{\star}$, to a vertical line $x_{\star}=1$ in the plane $y_{\star}=0$, and to inclined lines of angle $\arccos \left(1 / x_{*}\right)$ to the vertical in planes of constant $x_{\star}$. This is just the structure described by Crapper (1959), Miles (1971) and Sturova (1974, 1980).

\subsection{Steady approach}

Two features of the wave field are missed by the preceding analysis: the presence of a static disturbance centred on the source, extending both ahead of and behind it, and which in the literature has been given such various names as 'non-wave term' (Crapper 1959), 'near field' (Miles 1971), 'upstream effect' (Sturova 1974), 'upstream propagating waves' (Redekopp 1975), 'potential flow' (Gilreath \& Brandt 1985), 'cyclonic disturbance' (Cheng, Hefazi \& Brown 1984; Hefazi \& Cheng 1988); for an extended source, the spanwise confinement of the waves to a strip of the source width, and whose existence, revealed by theory (Crapper 1959, 1962), has been confirmed by both experiments (Castro 1987) and simulations (Hanazaki 1988; Suzuki \& Kuwahara 1992). Here the steady analysis of $\S 4$ is seen to provide explanations for these phenomena.

Our starting point is, from the substitution of $I(5.10)$ in (4.5),

$$
\frac{\partial \psi}{\partial t}(\boldsymbol{r}, t)=\frac{m_{0}}{2 \pi^{2} U} \operatorname{Im} f_{0}^{\infty} K_{0}\left[\left(k_{x}^{2} r_{\perp}^{2}-\frac{N^{2}}{U^{2}} z^{2}\right)^{\frac{1}{2}}\right] \frac{\mathrm{e}^{-i k_{x} x_{1}}}{\left(k_{x}^{2}-N^{2} / U^{2}\right)^{\frac{1}{2}}} \mathrm{~d} k_{x},
$$


(a)

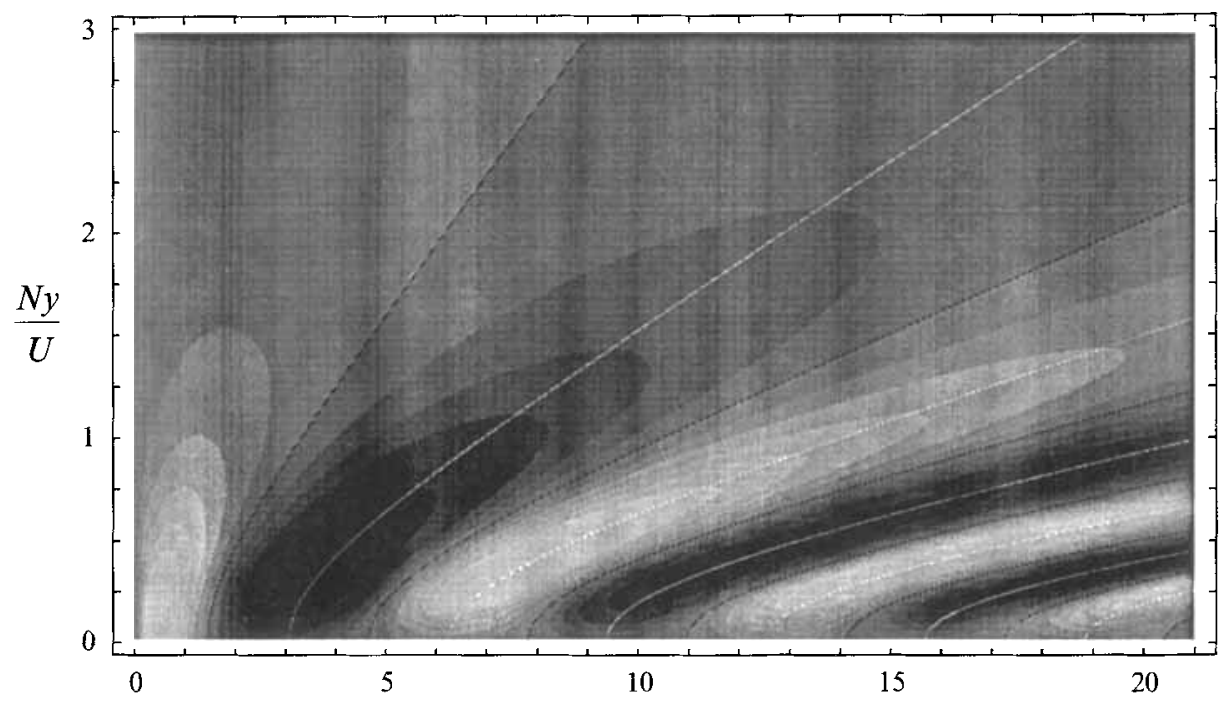

(b)
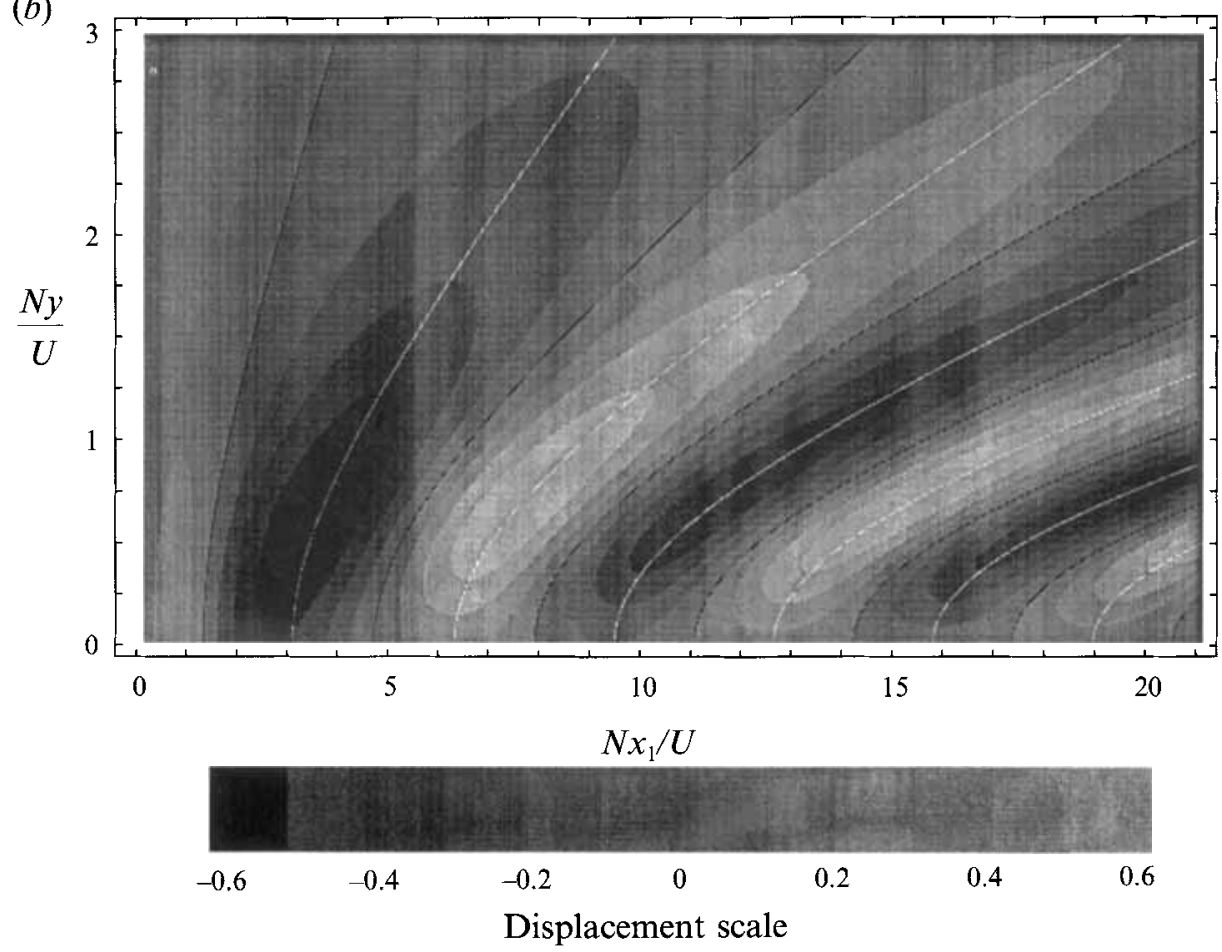

FIGURE 7. Normalized vertical displacement $\left(2 \pi U^{3} / N^{2} m_{d}\right) \zeta$ in the planes $(a) N z / U=\frac{1}{2}$, (b) $N z / U=1$. Only the part $y \geqslant 0$ of the displacement field, symmetric about the $x_{1}$-axis, is shown; black lines are nodal lines, white lines are crests and throughs.

which is equivalent to formulae (62) of Crapper (1959), (6.23) of Miles (1971) and (3.15) of Umeki \& Kambe (1989), and in the planes $y=0$ and $z=0$ is reduced through straightforward changes of variables to formulae (10)-(11) of Gorodtsov \& Teodorovich (1980). On the coordinates axes this is readily evaluated, using 
Gradshteyn \& Ryzhik (1980), as

$$
\begin{aligned}
& \left.\frac{\partial \psi}{\partial t}\right|_{y=z=0}=-\frac{m_{0}}{4 \pi U} S_{0,0}\left(\frac{N}{U}\left|x_{1}\right|\right) \quad\left(x_{1}<0\right) \\
& =\infty \quad\left(x_{1}>0\right), \\
& \left.\frac{\partial \psi}{\partial t}\right|_{x_{1}=z=0}=-\frac{m_{0}}{4 \pi U} I_{0}\left(\frac{N}{2 U}|y|\right) K_{0}\left(\frac{N}{2 U}|y|\right), \\
& \left.\frac{\partial \psi}{\partial t}\right|_{x_{1}=y=0}=\frac{m_{0}}{8 U} J_{0}\left(\frac{N}{2 U}|z|\right) Y_{0}\left(\frac{N}{2 U}|z|\right),
\end{aligned}
$$

and becomes asymptotically

$$
\begin{array}{lr}
\left.\frac{\partial \psi}{\partial t}\right|_{y=z=0} \sim-\frac{m_{0}}{4 \pi N\left|x_{1}\right|} \quad\left(x_{1}<0\right. \text { and } & \left.\frac{N}{U}\left|x_{1}\right| \gg 1\right), \\
\left.\frac{\partial \psi}{\partial t}\right|_{x_{1}=z=0} \sim-\frac{m_{0}}{4 \pi N|y|} & \left(\frac{N}{U}|y| \gg 1\right), \\
\left.\frac{\partial \psi}{\partial t}\right|_{x_{1}=y=0} \sim-\frac{m_{0}}{4 \pi N|z|} \cos \left(\frac{N}{U}|z|\right) & \left(\frac{N}{U}|z| \gg 1\right),
\end{array}
$$

where $J_{0}$ and $Y_{0}$ are Bessel functions of the first and second kind, respectively, $I_{0}$ and $K_{0}$ are modified Bessel functions of the first and second kind, respectively, $\mathbf{H}_{0}$ is a Struve function and $S_{0,0}=\frac{1}{2} \pi\left(\mathbf{H}_{0}-Y_{0}\right)$ is a Lommel function. This not only indicates coherence with expansion (5.7) in the plane $x_{1}=0$, the necessary factor $\frac{1}{2}$ being taken into account, but, more importantly, confirms both the divergence of the internal potential on the source path $\left(r_{\perp}=0, x_{1}>0\right)$ and the presence of a non-wave disturbance upstream of it.

The exact form of this disturbance is obtained, as did Crapper (1959) for internal waves and Miles $(1969 a)$ for inertial waves, by deforming the integration path of (5.14) as shown in figure 8. The upstream disturbance is identified as the integral along the imaginary axis, namely

$$
\frac{\partial \psi_{u}}{\partial t}(\boldsymbol{r}, t)=\frac{m_{0}}{4 \pi U} \operatorname{sgn} x_{1} \int_{0}^{\infty} J_{0}\left[\left(\chi^{2} r_{\perp}^{2}+\frac{N^{2}}{U^{2}} z^{2}\right)^{\frac{1}{2}}\right] \frac{\mathrm{e}^{-\chi\left|x_{1}\right|}}{\left(\chi^{2}+N^{2} / U^{2}\right)^{\frac{1}{2}}} \mathrm{~d} \chi,
$$

and in a homogeneous fluid is associated with a velocity potential $\partial^{2} \psi_{u} / \partial t^{2}$ identical to that, $-m_{0} /\left(4 \pi r_{1}\right)$, for irrotational flow. Thus, consistently with Gilreath \& Brandt (1985), the upstream disturbance represents the 'potential flow', modified by the stratification, around the source of the waves.

Its asymptotic form follows from the application of Watson's lemma to (5.21), but for a complete picture of the wave field it is more adequate to write (5.14) as the inverse Fourier transform, of argument $x_{1}$,

$$
\frac{\partial \psi}{\partial t}(\boldsymbol{r}, t)=-\frac{m_{0}}{\pi U} \operatorname{Im} \mathrm{FT}^{-1}\left\{\frac{H\left(k_{x}\right)}{\left(k_{x}^{2}-N^{2} / U^{2}\right)^{\frac{1}{2}}} K_{0}\left[\left(k_{x}^{2} r_{\perp}^{2}-\frac{N^{2}}{U^{2}} z^{2}\right)^{\frac{1}{2}}\right]\right\},
$$

and to evaluate this transform for $\left|x_{1}\right| \rightarrow \infty$ and fixed $r_{\perp}$, that is in the vicinity of the $x_{1}$-axis, by Lighthill's rule $(1958, \S 4.3)$. For convenience the same definition has for the moment been adopted for transforms with respect to $x_{1}$ as for transforms with 


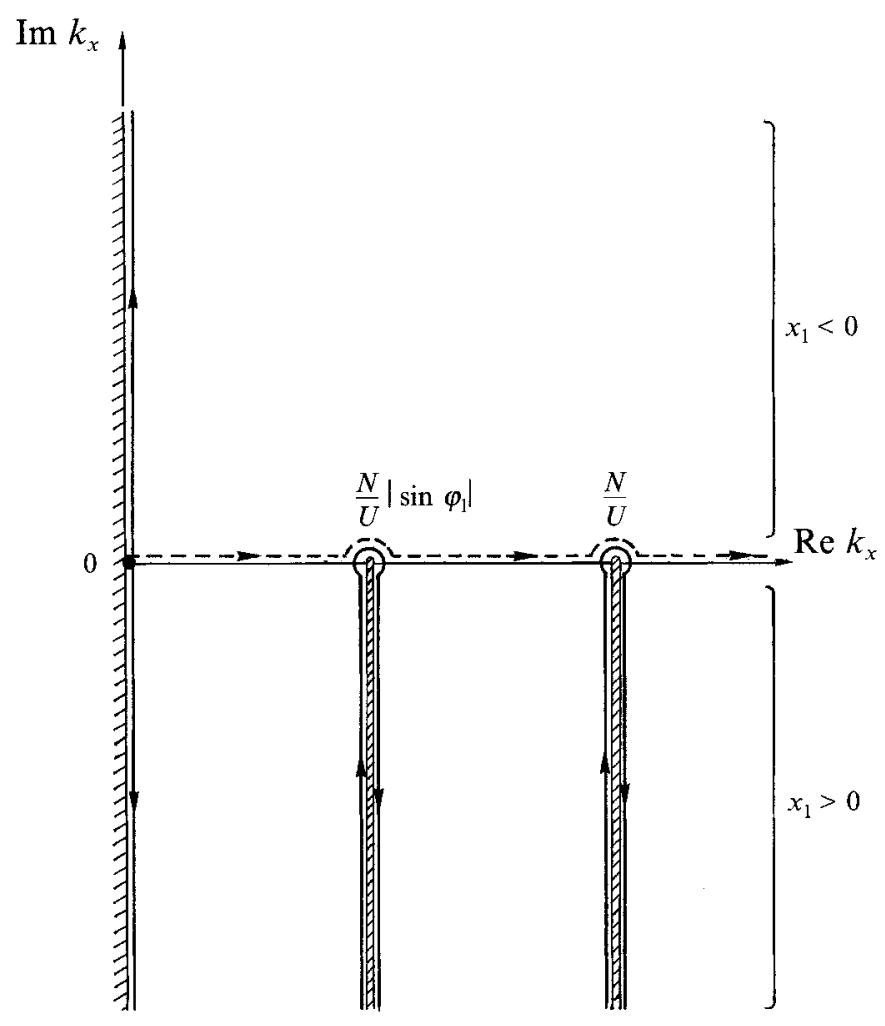

FIGURE 8. Separation of the upstream disturbance: ---, original integration path; - deformed integration path.

respect to $t$. Then, adding up the contributions of the singularities $0,(N|z|) /\left(U r_{\perp}\right)$ and $N / U$ of the original function and using (A 1)-(A 3) in Appendix A, we find, for $N\left|x_{1}\right| / U \gg 1$ and fixed $N r_{\perp} / U$,

$$
\begin{aligned}
\frac{\partial \psi}{\partial t}(r, t) \sim & \frac{m_{0}}{4 \pi U} \frac{U}{N x_{1}} J_{0}\left(\frac{N}{U}|z|\right) \\
& -H\left(x_{1}\right) \frac{m_{0}}{2 \pi U} \frac{U}{N x_{1}} \frac{r_{\perp}}{|y|} \cos \left[\frac{N}{U}|z|\left(\frac{x_{1}}{r_{\perp}}+\frac{r_{\perp}}{2 x_{1}}\right)\right] \\
& -H\left(x_{1}\right) \frac{m_{0}}{\pi U}\left(\frac{U}{2 \pi N x_{1}}\right)^{\frac{1}{2}} K_{0}\left(\frac{N}{U}|y|\right) \cos \left(\frac{N}{U} x_{1}-\frac{\pi}{4}\right),
\end{aligned}
$$

namely a decomposition of internal waves into the upstream disturbance, the permanent gravity waves, of frequency $N|z| / r_{\perp}$, given by (5.13), and permanent buoyancy oscillations of frequency $N$, respectively. This is just one of the results of Crapper (1959).

Consistency with $\S 5.1$ requires its generalization in the whole of the far field $r_{1} \rightarrow \infty$ with $\theta_{1}$ and $\varphi_{1}$ fixed. Following a procedure applied by Miles $(1969 a)$ to inertial waves, we replace for $\left|x_{1}\right| \rightarrow \infty$ and fixed $r_{\perp} /\left|x_{1}\right|$ the modified Bessel function 


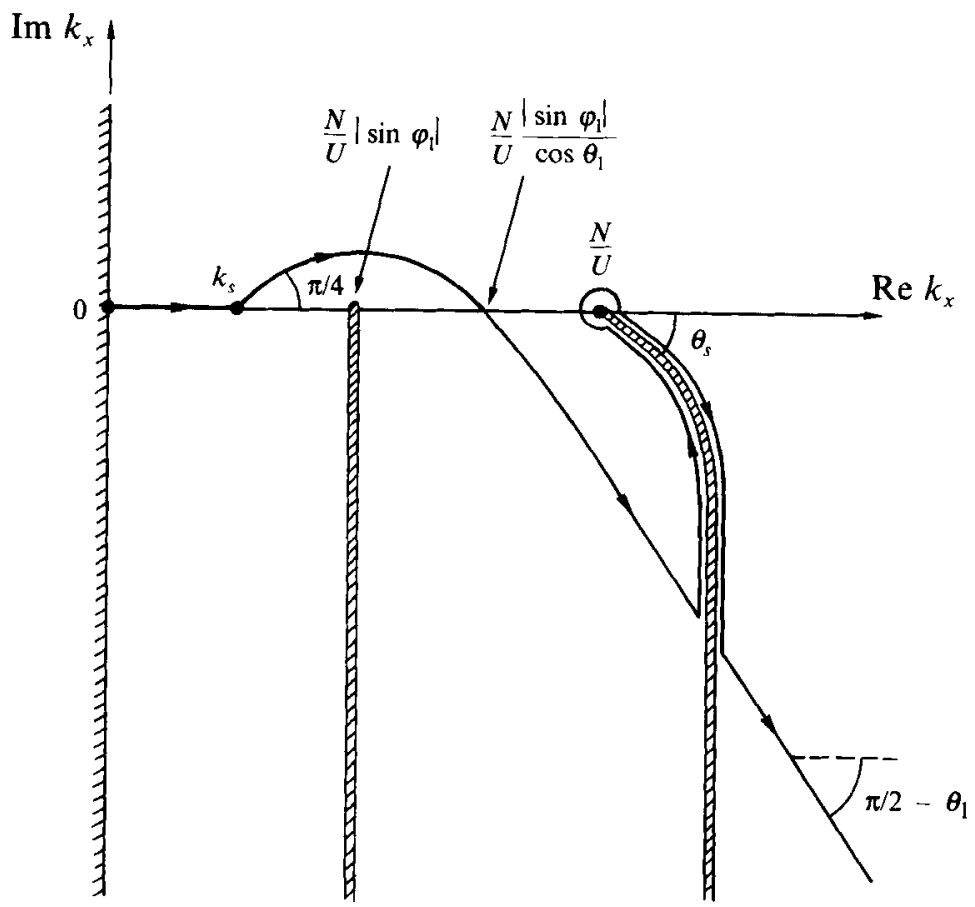

FIGURE 9. Deformation of contour for the far-field evaluation of the waves, for $x_{1}>0$ and $\cos \theta_{1}>\left|\sin \varphi_{1}\right|$.

$K_{0}$ in (5.14) by its asymptotic expansion, so that

$$
\frac{\partial \psi}{\partial t}(\boldsymbol{r} ; t) \sim \frac{m_{0}}{(2 \pi)^{\frac{3}{2}} U\left(\boldsymbol{r}_{1} \sin \theta_{1}\right)^{\frac{1}{2}}} \operatorname{Im} f_{0}^{\infty} \frac{\mathrm{e}^{r_{1} \phi\left(k_{x}\right)}}{\left(k_{x}^{2}-\frac{N^{2}}{U^{2}}\right)^{\frac{1}{2}}\left(k_{x}^{2}-\frac{N^{2}}{U^{2}} \sin ^{2} \varphi_{1}\right)^{\frac{1}{4}}} \mathrm{~d} k_{x},
$$

with

$$
\phi\left(k_{x}\right)=-\mathrm{i} k_{x} \cos \theta_{1}-\left(k_{x}^{2}-\frac{N^{2}}{U^{2}} \sin ^{2} \varphi_{1}\right)^{\frac{1}{2}} \sin \theta_{1},
$$

and we evaluate this integral asymptotically by a combination of the methods of stationary phase and of steepest descents (Bleistein 1984, $\$ 2.7$ and 7.2-8.1). The corresponding deformation of contour is shown in figure 9. For $x_{1}>0$ the integrand has a saddle point $k_{s}=(N / U) \cos \theta_{1}\left|\sin \varphi_{1}\right|$, and the deformed integration path follows the real axis from the endpoint 0 to $k_{s}$, then continues along the path of steepest descent emanating from $k_{s}$, and then, if $\cos \theta_{1}>\left|\sin \varphi_{1}\right|$, crosses the cut emanating from the branch point $N / U$. In that case three contributions arise, associated with the three critical points $0, k_{s}$ and $N / U$ of the integrand; a preliminary deformation of the cut is required, to transform it locally into a path of steepest descent making the angle $\theta_{s}=-\arctan \left(\cot \theta_{1}\left|\cos \varphi_{1}\right|\right)$ with the real axis. For $x_{1}=0$ the endpoint and the saddle point merge, and define a path of steepest descent parallel to the real axis at infinity. For $x_{1}<0$ there is no saddle point, and the only contribution to (5.24) comes from the endpoint.

Straightforward but lengthy calculations yield, then, for $N r_{1} / U \gg 1$ and fixed $\theta_{1}$, 
$\varphi_{1}$,

$$
\begin{aligned}
\frac{\partial \psi}{\partial t}(\boldsymbol{r}, t) \sim & \frac{m_{0}}{2 \pi N x_{1}}\left(\frac{U}{2 \pi N|z|}\right)^{\frac{1}{2}} \cos \left(\frac{N}{U}|z|-\frac{\pi}{4}\right) \\
& -H\left(x_{1}\right) \frac{m_{0}}{2 \pi N r_{1}} \frac{1}{\left(\sin ^{2} \theta_{1}+\cos ^{2} \theta_{1} \cos ^{2} \varphi_{1}\right)^{\frac{1}{2}}} \cos \left(\frac{N}{U} r_{1}\left|\sin \varphi_{1}\right|\right) \\
& -H\left(x_{1}\right) \frac{m_{0}}{2 \pi N\left(x_{1} r_{\perp}\right)^{\frac{1}{2}}} \exp \left(-\frac{N}{U}|y|\right) \frac{H\left(\cos \theta_{1}-\left|\sin \varphi_{1}\right|\right)}{\left(\sin ^{2} \theta_{1}+\cos ^{2} \theta_{1} \cos ^{2} \varphi_{1}\right)^{\frac{1}{4}}} \\
& \times \cos \left(\frac{N}{U} x_{1}-\frac{\pi}{4}-\frac{3}{2} \arctan \frac{\tan \theta_{1}}{\left|\cos \varphi_{1}\right|}\right),
\end{aligned}
$$

where for $x_{1}=0$ the third term is absent and the first two terms combine into one half of the second term. In this way the presence of the three components exhibited by (5.23) is confirmed, and in their common area of validity $N\left|x_{1}\right| / U \gg N r_{\perp} / U \gg 1$ both expansions coincide with each other.

For point sources, when physically meaningful quantities such as the vertical displacement $\zeta$ are considered, gravity waves are dominant. However, as the finite cross-section of any real source is taken into account, interference between the short waves originating at the various points of this source are especially important near its path, and blur gravity waves (Miles 1971; Janowitz 1984). This gives rise to the upstream disturbance and to buoyancy oscillations.

The upstream disturbance exhibits oscillations along the vertical, attributed by spectral analysis (i.e. Fourier transformation of the Bessel function $J_{0}(N|z| / U)$ ) or study of the wavenumber surface (Redekopp 1975) to waves of zero frequency and vertical wavenumbers $k_{z}$ ranging from 0 to $N / U$ and concentrated near $N / U$. Lighthill $(1978, \S 4.12)$ explained how such waves can be found upstream: since their horizontal wavenumber $k_{h}$ is 0 , their group velocity along the $x_{1}$-axis is $\pm N /\left|k_{z}\right|$ and can be directed upstream and exceed the velocity $U$ of the source. The upstream disturbance is, thus, of the same kind as the columnar disturbances known to arise in two-dimensional flow of unbounded fluids (Baines 1987) and three-dimensional flow of fluids of finite depth (Castro \& Snyder 1988; Hanazaki 1989), and to be associated with the blocking of the flow by the stratification, but never before registered in threedimensional flow of unbounded fluids; even if, unlike these disturbances, the upstream disturbance decreases with $\left|x_{1}\right|$ and is then centred on $x_{1}=0$. For an extended source it should appear as a maximum centred on point $O_{1}$ and superimposed on the wavecrests associated with gravity waves. The calculations of Smith (1980), confirmed by both simulations (Smolarkiewicz \& Rotunno 1989; Rotunno \& Smolarkiewicz 1991) and observations (Umeki \& Kambe 1989), exhibit how, as the streamwise dimension of the source increases from 0 to $O(U / N)$, successive wavecrests vanish and give way to that maximum.

Buoyancy oscillations originate from the second term of integral (3.8), eliminated in (3.12) by the application of the method of stationary phase (see Appendix B). They decrease exponentially with the scaled spanwise distance $N|y| / U$, and thus are confined to a strip approximately one wavelength wide. For an extended source the scaling distance is modified, but the decrease remains essentially exponential and spanwise. Then, for certain source shapes, buoyancy oscillations can dominate gravity waves and be confined to a strip of approximately the source width, consistently with Crapper (1959, 1962), Castro (1987), Hanazaki (1988) and Suzuki \& Kuwahara (1992). 


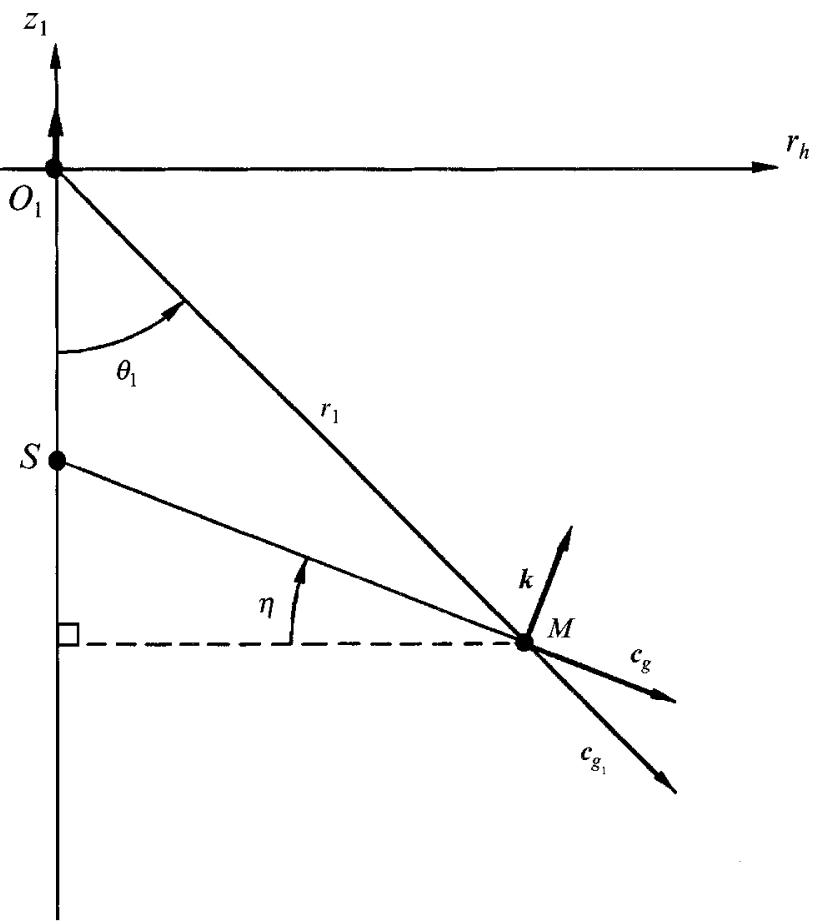

FIGURE 10. Geometry for internal wave radiation by a steady source in uniform vertical motion.

\section{Uniform vertical motion of a steady source}

We consider now the uniform vertical motion of a steady source, a problem which again has meteorological applications - connected to the rise of thermals (Warren $1960)$. In the moving system of coordinates $\left(x, y, z_{1}\right)$, with the $z_{1}$-axis directed vertically upwards, we use both cylindrical coordinates $\left(r_{h}, z_{1}\right)$ and spherical coordinates $\left(r_{1}, \theta_{1}\right)$, defined by

$$
r_{h}=\left(x^{2}+y^{2}\right)^{\frac{1}{2}}=r_{1} \sin \theta_{1}, \quad z_{1}=z-U t=-r_{1} \cos \theta_{1},
$$

and represented in figure 10 . The velocity of the source is $v_{0}=U \boldsymbol{e}_{z}$, with $\boldsymbol{e}_{z}$ a unit vector along the $z_{1}$-axis, and points either upwards $(U>0)$ or downwards $(U<0)$.

The description of the waves requires the introduction of an additional variable, which we choose as the angle $\eta$ of the direction of propagation with the horizontal. The distance between emission and reception is, in cylindrical components,

$$
\boldsymbol{R}\left(\tau_{s}\right)=\frac{r_{h}}{\cos \eta}[\cos \eta,-\sin \eta]
$$

so that the retarded time equation becomes a cubic equation for $\tan \eta$, namely

$$
\tan ^{3} \eta+2 \tan \eta-\cot \theta_{1}=0
$$

whose real solution is

$$
\tan \eta=\left[\left(\frac{\cot ^{2} \theta_{1}}{4}+\frac{8}{27}\right)^{\frac{1}{2}}+\frac{\cot \theta_{1}}{2}\right]^{\frac{1}{3}}-\left[\left(\frac{\cot ^{2} \theta_{1}}{4}+\frac{8}{27}\right)^{\frac{1}{2}}-\frac{\cot \theta_{1}}{2}\right]^{\frac{1}{3}}
$$




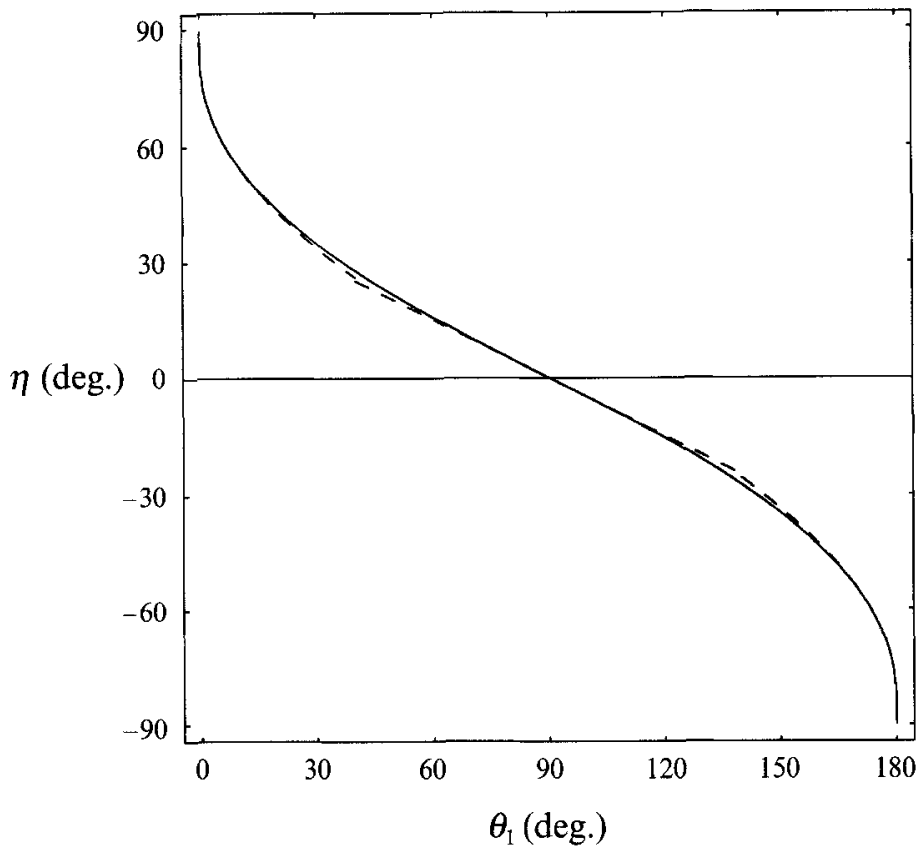

FIGURE 11. Solution of equation (6.3) for the angular variable $\eta$ : - exact result (6.4);---, asymptotic results, deduced from (6.12) and (6.14), $\eta \sim \frac{1}{2} \pi-\theta_{1}^{\frac{1}{3}}-\frac{1}{3} \theta_{1}$ for $\theta_{1} \rightarrow 0, \eta \sim \frac{1}{2}\left(\frac{1}{2} \pi-\theta_{1}\right)$ for $\theta_{1} \rightarrow \frac{1}{2} \pi$ and $\eta \sim-\frac{1}{2} \pi+\left(\pi-\theta_{1}\right)^{\frac{1}{3}}+\frac{1}{3}\left(\pi-\theta_{1}\right)$ for $\theta_{1} \rightarrow \pi$.

in terms of which $\tau_{s}$ is given by

$$
t-\tau_{s}=\frac{r_{h}}{U} \frac{\sin \eta}{\cos ^{3} \eta}
$$

and the causality condition $\tau_{s}<t$ imposes, for $U>0$, that $0<\theta_{1}<\frac{1}{2} \pi$ and $0<\eta<\frac{1}{2} \pi$, and, for $U<0$, that $\frac{1}{2} \pi<\theta_{1}<\pi$ and $-\frac{1}{2} \pi<\eta<0$. This means that all the waves generated since the beginning of the motion are found downstream (i.e. that $\left.U z_{1}<0\right)$, and that those generated at any particular time are situated behind the position $S$ of the source at that time (cf. figure 10). A plot of $\eta$ versus $\theta_{1}$ is given in figure 11.

In terms of $\eta$ we obtain, then, the frequency and wavelength

$$
\omega=N|\sin \eta|, \quad \lambda=2 \pi \frac{U}{N} \cot \eta,
$$

the group velocities in the fixed and moving reference frames

$$
\begin{gathered}
c_{g}=U \frac{\cos ^{2} \eta}{\sin \eta}[\cos \eta,-\sin \eta], \\
c_{g_{1}}=c_{g}-U e_{z}=U \frac{\left(1+\cos ^{2} \eta \sin ^{2} \eta\right)^{\frac{1}{2}}}{\sin \eta}\left[\sin \theta_{1},-\cos \theta_{1}\right],
\end{gathered}
$$

and the far-field velocity, for $N r_{1} /|U| \gg 1$,

$$
\boldsymbol{v}(\boldsymbol{r}, t) \sim H\left(-U z_{1}\right) \frac{N m_{0}}{2 \pi U r_{h}} \frac{\sin \eta \cos \eta}{\left(2+\sin ^{2} \eta\right)^{\frac{1}{2}}} \frac{\boldsymbol{R}}{R} \cos \left(\frac{N}{|U|} r_{h} \frac{\sin ^{2} \eta}{\cos ^{3} \eta}\right),
$$




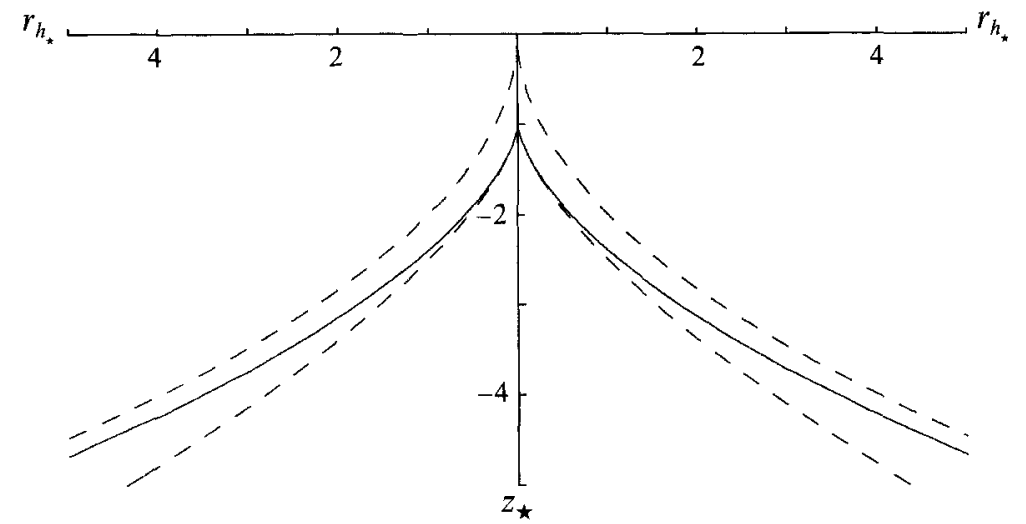

FIGURE 12. Surfaces of constant phase for uniform vertical motion of a steady source: surfaces (6.11);---, asymptotic surfaces $z_{\star}^{2} \sim 4 r_{h_{\star}}$ and $\left|z_{\star}\right| \sim 1+\frac{3}{2} r_{h_{\star}}^{\frac{2}{3}}$, deduced from (6.13) and (6.15).

consistently with Warren (1960) and, up to misprints, with Makarov \& Chashechkin (1982). Only the frequency is bounded, and none of the group velocities is transverse.

The two forms of the phase, deduced from each other by (6.3),

$$
\Phi=\frac{N}{|U|} r_{h} \frac{\sin ^{2} \eta}{\cos ^{3} \eta}=-\frac{N}{|U|} z_{1} \frac{\sin \eta}{1+\cos ^{2} \eta}
$$

imply that surfaces of constant phase have, in terms of $r_{\star}=\left(N r_{1}\right) /(|U| \Phi)$, the parametric equation

$$
r_{h_{\star}}=\frac{\cos ^{3} \eta}{\sin ^{2} \eta}, \quad z_{\star}=-\frac{1+\cos ^{2} \eta}{\sin \eta}
$$

and thus have the 'flared skirt' or 'herring bone' shape shown in figure 12, with cusps on the source path at $z_{\star}=-\operatorname{sgn} U$ and parabolic branches $z_{\star}^{2} \sim 4 r_{h_{\star}}$ far from it. This agrees with Lighthill $(1967,1978, \S 4.12)$ and Mowbray \& Rarity (1967), and is reflected in the structure of the velocity field, studied by Warren (1960) and visualized by Stevenson et al. (1983), Gärtner (1983a,b) and Gärtner et al. (1986).

In two regions this analysis breaks down: close to the source path, where shorter waves are found and the point source model is invalidated, with, for $N\left|z_{1}\right| /|U| \gg N r_{h} /|U| \gg 1$,

$$
\eta \sim\left[\frac{\pi}{2}-\left(\frac{r_{h}}{\left|z_{1}\right|}\right)^{\frac{1}{3}}-\frac{1}{3} \frac{r_{h}}{\left|z_{1}\right|}\right] \operatorname{sgn} U
$$

so that $\quad \boldsymbol{v}(\boldsymbol{r}, t) \sim-H\left(-U z_{1}\right) \frac{N m_{0}}{2 \pi \sqrt{3} U} \frac{\boldsymbol{e}_{z}}{r_{h}^{\frac{2}{3}}\left|z_{1}\right|^{\frac{1}{3}}} \cos \left[\frac{N}{|U|}\left(\left|z_{1}\right|-\frac{3}{2} r_{h}^{\frac{2}{3}}\left|z_{1}\right|^{\frac{1}{3}}\right)\right]$

close to the source level, where longer waves are found and the Boussinesq approximation is invalidated, with, for $N r_{h} /|U| \gg N\left|z_{1}\right| /|U| \gg 1$,

$$
\eta \sim-\frac{1}{2} \frac{z_{1}}{r_{h}}
$$

so that

$$
\boldsymbol{v}(\boldsymbol{r}, t) \sim-H\left(-U z_{1}\right) \frac{N m_{0}}{4 \pi \sqrt{2} U} \frac{z_{1}}{r_{h}^{2}} \frac{\boldsymbol{r}_{h}}{r_{h}} \cos \left(\frac{N}{|U|} \frac{z_{1}^{2}}{4 r_{h}}\right)
$$


Both limiting forms are shown in figures 11 and 12. They describe the cusps and parabolic branches of the wavecrests, respectively, and coincide, up to misprints, with the results of Grigor'ev \& Dokuchaev (1970).

As before, we can think of the analysis of $\S 4$ as a source of complementary insight into the waves. The substitution of I (5.8) in (4.5) yields, consistently with formulae (18) of Warren (1960) and (34) of Grigor'ev \& Dokuchaev (1970),

$$
\psi(\boldsymbol{r}, t)=\frac{m_{0}}{2 \pi^{2} U^{2}} \operatorname{Re} f_{0}^{\infty} K_{0}\left[\frac{k_{z}^{2} r_{h}}{\left(k_{z}^{2}-N^{2} / U^{2}\right)^{\frac{1}{2}}}\right] \frac{\mathrm{e}^{-i k_{z} z_{1} \operatorname{sgn} U}}{k_{z}^{2}-N^{2} / U^{2}} \mathrm{~d} k_{z} .
$$

By the same procedure as in $\S 5.2$, this proves that the asymptotic wave field combines the preceding gravity waves with a second wave term and an upstream disturbance. However, this second term is not a buoyancy oscillation but an evanescent gravity wave decreasing exponentially with the assumed large parameter, while the upstream disturbance reduces to exactly the same flow as in a homogeneous fluid, since no physical mechanism is susceptible of blocking a vertical stratified flow. Hence, no significant feature of the waves is missed by the analysis of $\S 3$.

\section{Uniform horizontal motion of an oscillating source}

We consider next the uniform horizontal motion of a source of purely oscillatory strength $\left(\omega_{0} \neq 0\right.$ and $m_{0}=$ const), examples being bodies which both translate and heave (Rehm \& Radt 1975), and turbulent wakes (Gilreath \& Brandt 1985). Studies of this problem (Stevenson \& Thomas 1969; Redekopp 1975; Rehm \& Radt 1975; Peat \& Stevenson 1975) have focused on the shape of surfaces of constant phase, and on the existence of several wave systems, most of which confined within caustics. Here for the first time an analytical approach of these points is proposed, including the justification of an approximate result cited, but not proved, by Gilreath \& Brandt (1985).

In the system of coordinates of $\S 5$, we introduce the reduced Doppler frequency

$$
\xi_{ \pm}=\frac{\omega_{ \pm}}{N}=\frac{\omega_{0}-U k_{x_{ \pm}}}{N}= \pm \frac{|z|}{\left(X_{ \pm}^{2}+r_{\perp}^{2}\right)^{\frac{1}{2}}}
$$

positive for sum waves, negative for difference waves, and for both of them satisfying $\left|\xi_{ \pm}\right|<\left|\sin \varphi_{1}\right|$. Denoting as $r=\omega_{0} / N$ the ratio of the frequency of the source to the frequency of the fluid, and deducing the sign of $X_{ \pm}$from the causality condition $\tau_{ \pm}<t$, we express the distance travelled by the waves between emission and reception as

$$
\boldsymbol{R}_{ \pm}=\frac{r_{\perp}}{\xi_{ \pm}}\left[\left(\sin ^{2} \varphi_{1}-\xi_{ \pm}^{2}\right)^{\frac{1}{2}} \operatorname{sgn}\left(\gamma-\xi_{ \pm}\right), \xi_{ \pm} \cos \varphi_{1}, \xi_{ \pm} \sin \varphi_{1}\right]
$$

and transform the retarded time equation into an equation for $\xi_{ \pm}$, namely

$$
\tan \theta_{1}=\frac{\xi_{ \pm}^{2}\left(\sin ^{2} \varphi_{1}-\xi_{ \pm}^{2}\right)^{\frac{1}{2}}}{r \sin ^{2} \varphi_{1}-\xi_{ \pm}^{3}} \operatorname{sgn}\left(r-\xi_{ \pm}\right),
$$

which, squared, yields the sixth-order necessary condition

$$
\xi_{ \pm}^{6}-\xi_{ \pm}^{4} \cos ^{2} \theta_{1} \sin ^{2} \varphi_{1}-2 r \xi_{ \pm}^{3} \sin ^{2} \theta_{1} \sin ^{2} \varphi_{1}+r^{2} \sin ^{2} \theta_{1} \sin ^{4} \varphi_{1}=0,
$$


in agreement with $\operatorname{Rehm} \& \operatorname{Radt}(1975)$, while $\tau_{ \pm}$itself is given in terms of $\xi_{ \pm}$by

$$
t-\tau_{ \pm}=\frac{r_{\perp}}{U} \frac{\left|\gamma-\xi_{ \pm}\right| \sin ^{2} \varphi_{1}}{\xi_{ \pm}^{2}\left(\sin ^{2} \varphi_{1}-\xi_{ \pm}^{2}\right)^{\frac{1}{2}}}
$$

For fixed $\theta_{1}$, the number and nature of the solutions of (7.3) follow from the behaviour of its right-hand side as a function of the variable $\xi_{\star}=\xi_{ \pm} /\left|\sin \varphi_{1}\right|$, with parameter $Y_{\star}=\Upsilon /\left|\sin \varphi_{1}\right|$, in the range $\left|\xi_{\star}\right|<1$ of admissible $\xi_{\star}$. This behaviour, illustrated in figure 13 , is determined by the position of $\xi_{ \pm}$with respect to $r$ and to the real roots, if any, of the third-order polynomial $\xi_{ \pm}^{3}-3 \gamma \xi_{ \pm}^{2}+2 \gamma \sin ^{2} \varphi_{1}$.

Difference waves are found downstream of the source, within the caustic

$$
\theta_{1}=\Theta_{-} \equiv \arctan \left[\frac{\Xi_{-}^{2}}{3 r\left(\sin ^{2} \varphi_{1}-\Xi_{-}^{2}\right)^{\frac{1}{2}}}\right],
$$

which corresponds to a maximum of the right-hand side of (7.3) at

$$
\begin{aligned}
\xi_{-}=\Xi_{-} & \equiv Y\left\{1-2 \cos \left[\frac{1}{3} \arccos \left(1-\frac{\sin ^{2} \varphi_{1}}{\Upsilon^{2}}\right)-\frac{\pi}{3}\right]\right\} \quad\left(\left|\sin \varphi_{1}\right|<\sqrt{ } 2 \Upsilon\right) \\
\equiv & \left\{1-\left[\frac{\sin ^{2} \varphi_{1}}{\Upsilon^{2}}-1+\left(\frac{\sin ^{2} \varphi_{1}}{\Upsilon^{2}}-2\right)^{\frac{1}{2}} \frac{\sin \varphi_{1}}{\Upsilon}\right]^{\frac{1}{3}}\right. \\
& \left.-\left[\frac{\sin ^{2} \varphi_{1}}{\Upsilon^{2}}-1-\left(\frac{\sin ^{2} \varphi_{1}}{\Upsilon^{2}}-2\right)^{\frac{1}{2}} \frac{\sin \varphi_{1}}{\Upsilon}\right]^{\frac{1}{3}}\right\} \quad\left(\left|\sin \varphi_{1}\right|>\sqrt{ } 2 \Upsilon\right),
\end{aligned}
$$

As Kelvin ship waves (Lighthill 1978, §3.10; Sharman \& Wurtele 1983), difference waves result from the superposition of a transverse wave system $\left(\Xi_{-}<\xi_{-}<0\right)$ and a divergent wave system $\left(-\left|\sin \varphi_{1}\right|<\xi_{-}<\Xi_{-}\right)$, named after the shape of their horizontal curves of constant phase (see figures 19 and 20 below), and which merge on the caustic.

The structure of sum waves is fairly different. Frequencies $0<\xi_{+}<\min \left(Y,\left|\sin \varphi_{1}\right|\right)$ are associated with 'downstream waves', which extend only behind the source and are confined within a wavefront defined for $\left|\sin \varphi_{1}\right|<\gamma$ by the caustic

$$
\theta_{1}=\Theta_{+}^{(d)} \equiv \arctan \left[\frac{\Xi_{+}^{2}}{3 \gamma\left(\sin ^{2} \varphi_{1}-\Xi_{+}^{2}\right)^{\frac{1}{2}}}\right] \quad\left(\left|\sin \varphi_{1}\right|<\gamma\right),
$$

corresponding to a maximum of the right-hand side of (7.3) at

$$
\xi_{+}=\Xi_{+} \equiv Y\left\{1-2 \cos \left[\frac{1}{3} \arccos \left(1-\frac{\sin ^{2} \varphi_{1}}{r^{2}}\right)+\frac{\pi}{3}\right]\right\} \quad\left(\left|\sin \varphi_{1}\right|<Y\right),
$$

and for $\left|\sin \varphi_{1}\right|>\Upsilon$ by the characteristic cone $\Upsilon=\left|z_{1}\right| / r_{1}=\sin \theta_{1}\left|\sin \varphi_{1}\right|$ or

$$
\theta_{1}=\Theta_{+}^{(d)} \equiv \arcsin \left(\frac{r}{\left|\sin \varphi_{1}\right|}\right) \quad\left(\left|\sin \varphi_{1}\right|>\Upsilon\right)
$$

corresponding to a change of the sign of the right-hand side of (7.3) at

$$
\xi_{+}=\Xi_{+} \equiv r \quad\left(\left|\sin \varphi_{1}\right|>r\right)
$$

By contrast, frequencies $\min \left(Y,\left|\sin \varphi_{1}\right|\right)<\xi_{+}<\left|\sin \varphi_{1}\right|$ are associated with 'upstream 

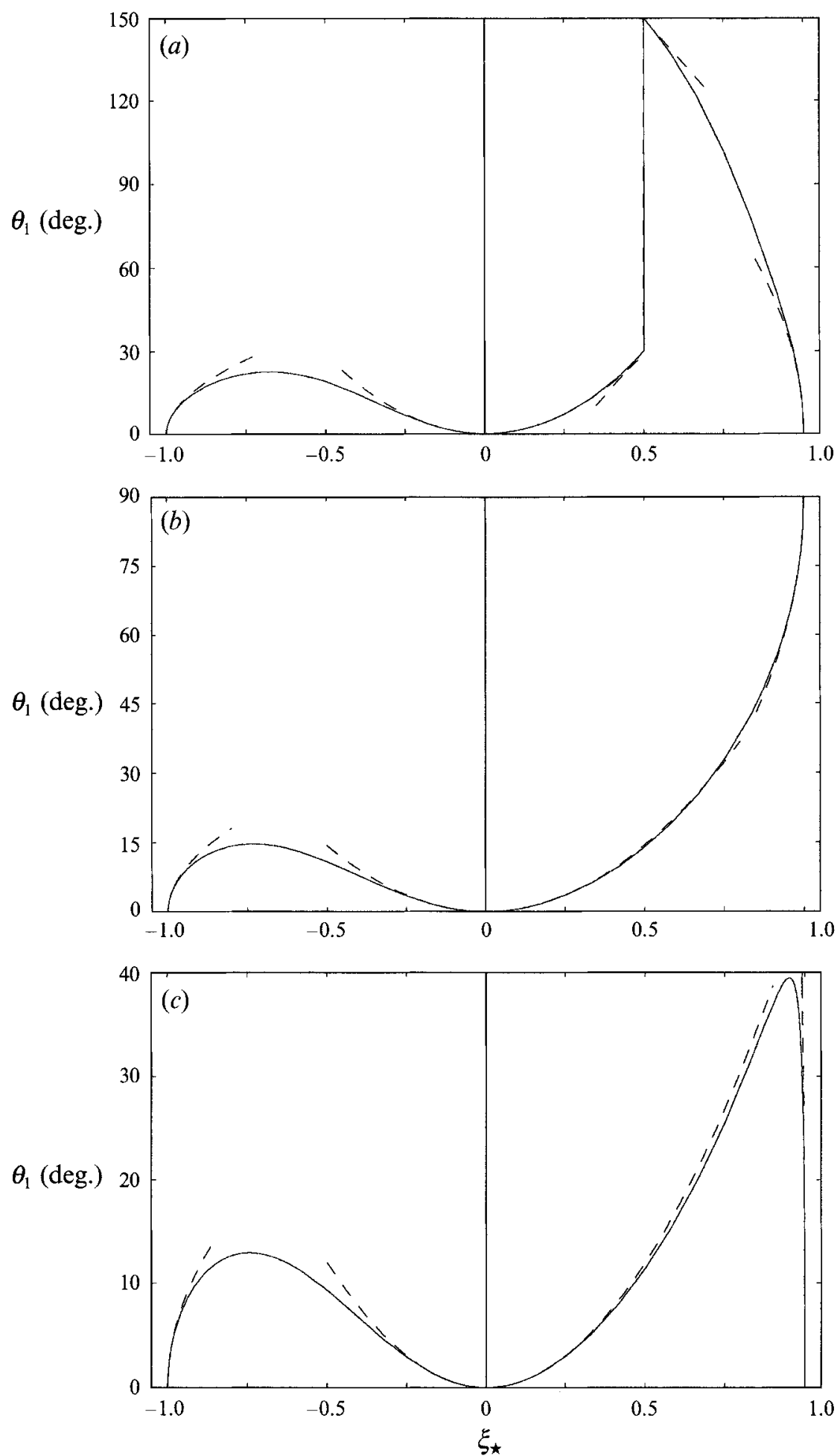

FIGURE 13. Solution of equation (7.3) for the frequency variable $\xi_{\star}$, for $(a) Y_{\star}=0.5,(b) Y_{\star}=1$, (c) $Y_{\star}=1.2:-$, exact result; ---, asymptotic results, deduced from (7.19), (7.21), (7.23) and (7.25), $\theta_{1} \sim \xi_{\star}^{2} / Y_{\star}$ for $\xi_{\star} \rightarrow 0, \theta_{1} \sim\left[2\left(1 \mp \xi_{\star}\right)\right]^{\frac{1}{2}} /\left|1 \mp Y_{\star}\right|$ for $\xi_{\star} \rightarrow \pm 1 \neq Y_{\star}, \theta_{1} \sim \Theta_{+}-2\left|Y_{\star}-\xi_{\star}\right| /\left(1-Y_{\star}^{2}\right)^{\frac{1}{2}}$ for $\xi_{\star} \rightarrow \Upsilon_{\star} \neq 1$ and $\theta_{1} \sim \frac{1}{2} \pi-3\left[\left(1-\xi_{\star}\right) / 2\right]^{\frac{1}{2}}$ for $\xi_{\star} \rightarrow 1=\Upsilon_{\star}$. 
waves', which extend on both sides of the source and are observed in the zone $\left|\sin \varphi_{1}\right|>r$, above or below a wavefront defined upstream by the characteristic cone

$$
\theta_{1}=\Theta_{+}^{(u)} \equiv \pi-\arcsin \left(\frac{r}{\left|\sin \varphi_{1}\right|}\right) \quad\left(\left|\sin \varphi_{1}\right|>\gamma\right),
$$

and downstream by the 'characteristic dihedron' $\gamma=\left|z_{1}\right| / r_{\perp}=\left|\sin \varphi_{1}\right|$; on the plane $x_{1}=0$,

$$
\left.\xi_{+}\right|_{x_{1}=0}=\left(\Upsilon \sin ^{2} \varphi_{1}\right)^{\frac{1}{3}}
$$

In both cases the transverse character of the waves for $0<\xi_{+}<\Xi_{+}$, and their divergent character for $\Xi_{+}<\xi_{+}<\left|\sin \varphi_{1}\right|$, are preserved.

Thus, for $Y<1$, difference waves, downstream sum waves and upstream sum waves are all present, while for $Y>1$ only the first two are observed. Whatever $Y$, they are confined within fronts, represented in figures 14 and 15, parts of which are caustics composed of pairs of cones symmetric with respect to the plane $z=0$, and of apex $O_{1}$ and axes in the plane $y=0$. As $Y$ increases the cones become progressively circular, until eventually, for $r \gg 1$,

$$
\Xi_{ \pm} \sim \pm\left(\frac{2}{3}\right)^{\frac{1}{2}}\left|\sin \varphi_{1}\right|, \quad \Theta_{ \pm} \sim \frac{2}{3^{\frac{3}{2}}} \frac{\left|\sin \varphi_{1}\right|}{r},
$$

as shown in figure 16; this agrees with the result, cited by Gilreath \& Brandt (1985), that for $Y \gg 1$, in the plane $y=0$, the waves are situated inside a wedge of apex $O_{1}$, axis $O_{1} x_{1}$ and semi-angle $\arctan (0.4 / \Upsilon)$.

It is, then, straightforward to derive the frequency and wavelength

$$
\omega_{ \pm}=N \xi_{ \pm}, \quad \lambda_{ \pm}=2 \pi \frac{U}{N}\left|\frac{\xi_{ \pm}}{\left(r-\xi_{ \pm}\right) \sin \varphi_{1}}\right|\left(\frac{\sin ^{2} \varphi_{1}-\xi_{ \pm}^{2}}{1-\xi_{ \pm}^{2}}\right)^{\frac{1}{2}},
$$

and the far-field velocity, for $N r_{1} / U \gg 1$,

$$
\begin{aligned}
& \boldsymbol{v}_{ \pm}(\boldsymbol{r}, t) \sim H\left(\Theta_{ \pm}-\theta_{1}\right) \frac{N m_{0}}{4 \pi U r_{\perp}}\left|\xi_{ \pm}\right|\left|\frac{\left(1-\xi_{ \pm}^{2}\right)\left(r-\xi_{ \pm}\right)}{\xi_{ \pm}^{3}-3 r \xi_{ \pm}^{2}+2 r \sin ^{2} \varphi_{1}}\right|^{\frac{1}{2}} \frac{\boldsymbol{R}_{ \pm}}{R_{ \pm}} \\
& \times \exp \left\{\mathrm{i}\left[\Phi_{ \pm} \pm \frac{\pi}{2} H\left(\left|\Xi_{ \pm}\right|-\left|\xi_{ \pm}\right|\right)\right]\right\},
\end{aligned}
$$

whose phase is written by (7.3) in either of the two forms

$$
\Phi_{ \pm}=\omega_{0} t-\frac{N}{U} x_{1} \frac{\left(\Upsilon-\xi_{ \pm}\right)^{2} \sin ^{2} \varphi_{1}}{\Upsilon \sin ^{2} \varphi_{1}-\xi_{ \pm}^{3}}=\omega_{0} t-\frac{N}{U} r_{\perp} \frac{\left(\Upsilon-\xi_{ \pm}\right)^{2} \sin ^{2} \varphi_{1}}{\xi_{ \pm}^{2}\left(\sin ^{2} \varphi_{1}-\xi_{ \pm}^{2}\right)^{\frac{1}{2}}} \operatorname{sgn}\left(\Upsilon-\xi_{ \pm}\right) .
$$

Surfaces of constant phase, defined as surfaces of constant $\Phi_{\star}=\left|\omega_{0} t-\Phi_{ \pm}\right|$, have in terms of $\boldsymbol{r}_{\star}=\left(N \boldsymbol{r}_{1}\right) /\left(U \Phi_{\star}\right)$ the parametric equation

$$
x_{\star}=\frac{r \sin ^{2} \varphi_{1}-\xi_{ \pm}^{3}}{\left(r-\xi_{ \pm}\right)^{2} \sin ^{2} \varphi_{1}} \operatorname{sgn}\left(r-\xi_{ \pm}\right), \quad r_{\perp *}=\frac{\xi_{ \pm}^{2}\left(\sin ^{2} \varphi_{1}-\xi_{ \pm}^{2}\right)^{\frac{1}{2}}}{\left(r-\xi_{ \pm}\right)^{2} \sin ^{2} \varphi_{1}} .
$$

They are shown in figures 17 and 18. In figures 19 and 20, obtained by Lighthill's method, they are intersected by horizontal and vertical planes, and reduce to the curves drawn by Stevenson \& Thomas (1969), Redekopp (1975), Rehm \& Radt (1975) and Peat \& Stevenson (1975).

In three cases this analysis breaks down. First, on caustics, expansion (7.16) 

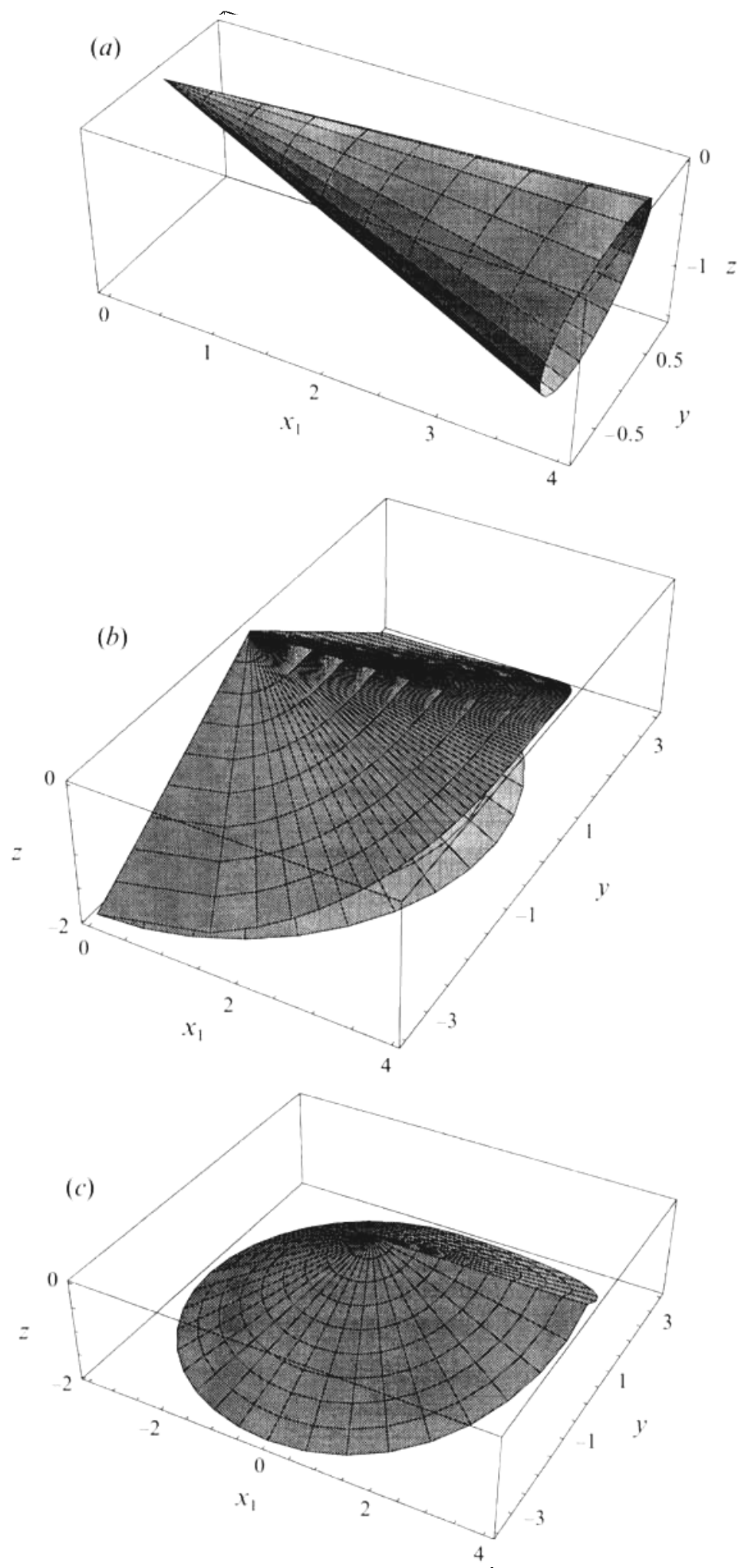

FIGURE 14. Wavefronts for an oscillating source in uniform horizontal motion, for $r=0.5$; $(a)$ difference waves, $(b)$ downstream sum waves, $(c)$ upstream sum waves. Only the lower half of the fronts, symmetric about the plane $z=0$, is shown. 

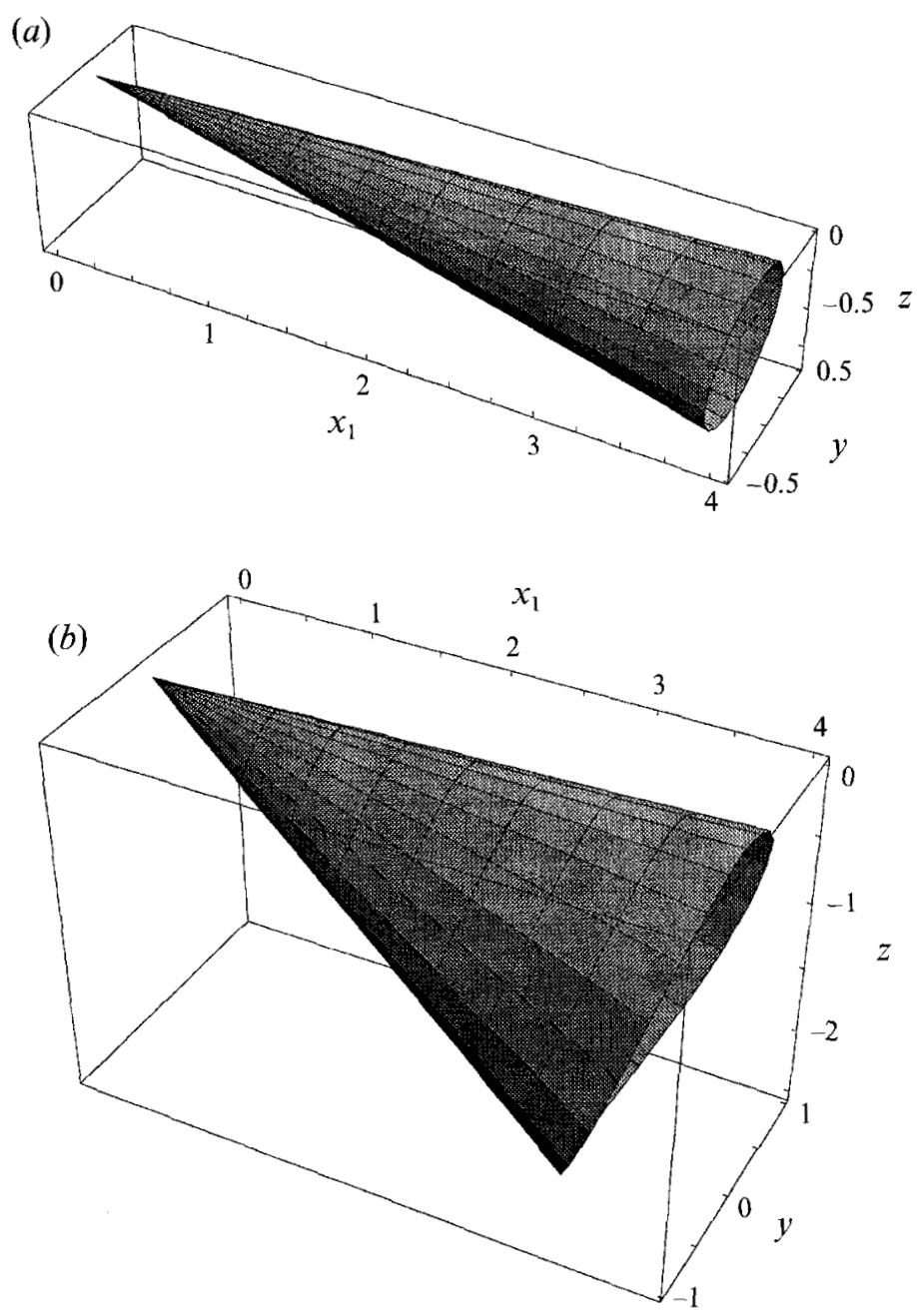

FIGURE 15. Wavefronts for an oscillating source in uniform horizontal motion, for $\gamma=1.2$; (a) difference waves, $(b)$ downstream sum waves.

predicts a divergence of the amplitude and a phase shift of $\frac{1}{2} \pi$ between divergent waves and transverse waves, as a consequence of the vanishing of the second derivative $A_{ \pm} \omega_{ \pm} c_{ \pm} / R_{ \pm}$of the phase (3.25). Lighthill $(1978, \S 4.11)$ explained how the method of stationary phase can be amended to overcome such local difficulties. This was not attempted.

The other two cases are, as in $\S \S 5$ and 6 above, places where $\lambda_{ \pm} \rightarrow 0$ and $\lambda_{ \pm} \rightarrow \infty$. Shorter waves are found near the source path, where $\xi_{ \pm}=0$ for transverse waves and $\xi_{ \pm}= \pm\left|\sin \varphi_{1}\right|$ for divergent waves. For $N x_{1} / U \gg N r_{\perp} / U \gg 1$, transverse waves satisfy

$$
\xi_{ \pm} \sim \pm\left(r \frac{|z|}{x_{1}}\right)^{\frac{1}{2}}
$$

so that

$$
\boldsymbol{v}_{ \pm}(r, t) \sim \mathrm{i} H\left(x_{1}\right) \frac{N m_{0}}{4 \pi \sqrt{ } 2 U}\left(\frac{r}{x_{1}|z|}\right)^{\frac{1}{2}} \boldsymbol{e}_{x} \exp \left\{\mathrm{i}\left[\omega_{0} t-\frac{N}{U} r x_{1} \pm 2 \frac{N}{U}\left(r x_{1}|z|\right)^{\frac{1}{2}}\right]\right\}
$$




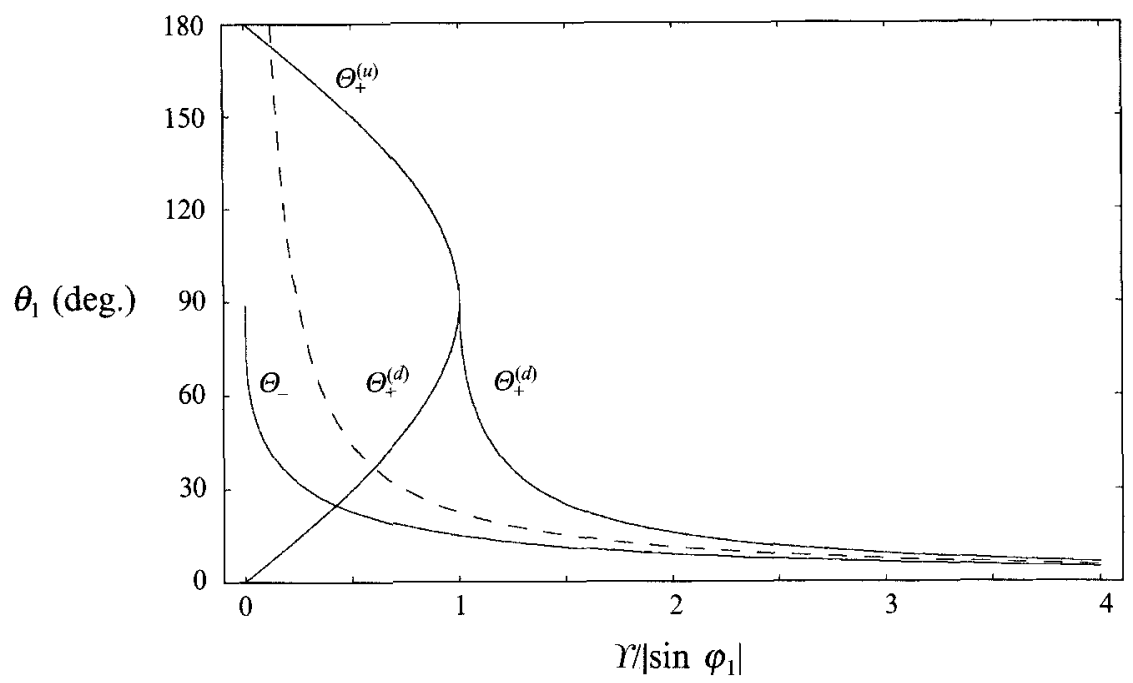

FIGURE 16. Equation of the wavefronts for an oscillating source in uniform horizontal motion: -, exact forms (7.6), (7.8), (7.10) and (7.12);---, asymptotic form (7.14).

which describes the contact at $x_{\star}=1 / Y$ of difference waves and downstream sum waves, along hyperbolic wavecrests. Similarly, for divergent waves, provided $\left|\sin \varphi_{1}\right| \neq$ $r$,

$$
\xi_{ \pm} \sim \pm \frac{|z|}{r_{\perp}} \mp \frac{1}{2}\left(1 \mp r \frac{r_{\perp}}{|z|}\right)^{2} \frac{r_{\perp}|z|}{x_{1}^{2}}
$$

so that

$$
\boldsymbol{v}_{ \pm}(\boldsymbol{r}, t) \sim H\left(x_{1}\right) \frac{N m_{0}}{4 \pi U} \frac{|y|}{r_{\perp}^{2}} \frac{\boldsymbol{r}_{\perp}}{r_{\perp}} \exp \left\{\mathrm{i}\left[\omega_{0} t-\frac{N}{U} x_{1}\left(r \mp \frac{|z|}{r_{\perp}}\right) \pm \frac{N}{U} \frac{r_{\perp}^{3}}{2 x_{1}|z|}\left(r \mp \frac{|z|}{r_{\perp}}\right)^{2}\right]\right\} .
$$

This describes for $y_{\star}=0$ the intersections at $x_{\star}=1 /|\gamma \mp 1|$ of difference waves and of sum waves with the source path, along elliptic or hyperbolic wavecrests, for $z_{\star} \rightarrow 0$ the contact at $x_{\star}=1 / \Upsilon$ of difference waves and downstream sum waves, and for $\left|\sin \varphi_{1}\right| \rightarrow Y$ the contact as $x_{\star} \rightarrow \infty$ of upstream sum waves and downstream sum waves. Note also the relationship between (5.13) and (7.22).

Longer waves are associated with sum waves and are found near the characteristic cone, where $\xi_{+}=r$. This defines a conical shell $N r_{1} / U \gg 1 /\left(\Theta_{+}-\theta_{1}\right) \gg 1$, which intersects the characteristic dihedron in the plane $\theta_{1}=\frac{1}{2} \pi$. Out of the intersection, i.e. for $\left|\sin \varphi_{1}\right| \neq \Upsilon$,

$$
\xi_{+} \sim r-\frac{1}{2} \Upsilon\left(\Theta_{+}-\theta_{1}\right) \cot \Theta_{+},
$$

so that

$$
\begin{aligned}
& \boldsymbol{v}_{+}(\boldsymbol{r}, t) \sim \mathrm{i} H\left(\Theta_{+}-\theta_{1}\right) \frac{\left(N^{2}-\omega_{0}^{2}\right)^{\frac{1}{2}} m_{0}}{8 \pi U r_{1}}\left(\frac{\Theta_{+}-\theta_{1}}{\sin \Theta_{+} \cos \Theta_{+}}\right)^{\frac{1}{2}} \frac{\boldsymbol{r}_{1}}{r_{1}} \\
& \times \exp \left\{\mathrm{i}\left[\omega_{0} t-\frac{\omega_{0}}{U} \frac{r_{1}}{4} \frac{\cos \Theta_{+}}{\sin ^{2} \Theta_{+}}\left(\Theta_{+}-\theta_{1}\right)^{2}\right]\right\},
\end{aligned}
$$

and the wavecrests are tangent to the characteristic cone as $r_{\star} \rightarrow \infty$. In the intersection, 

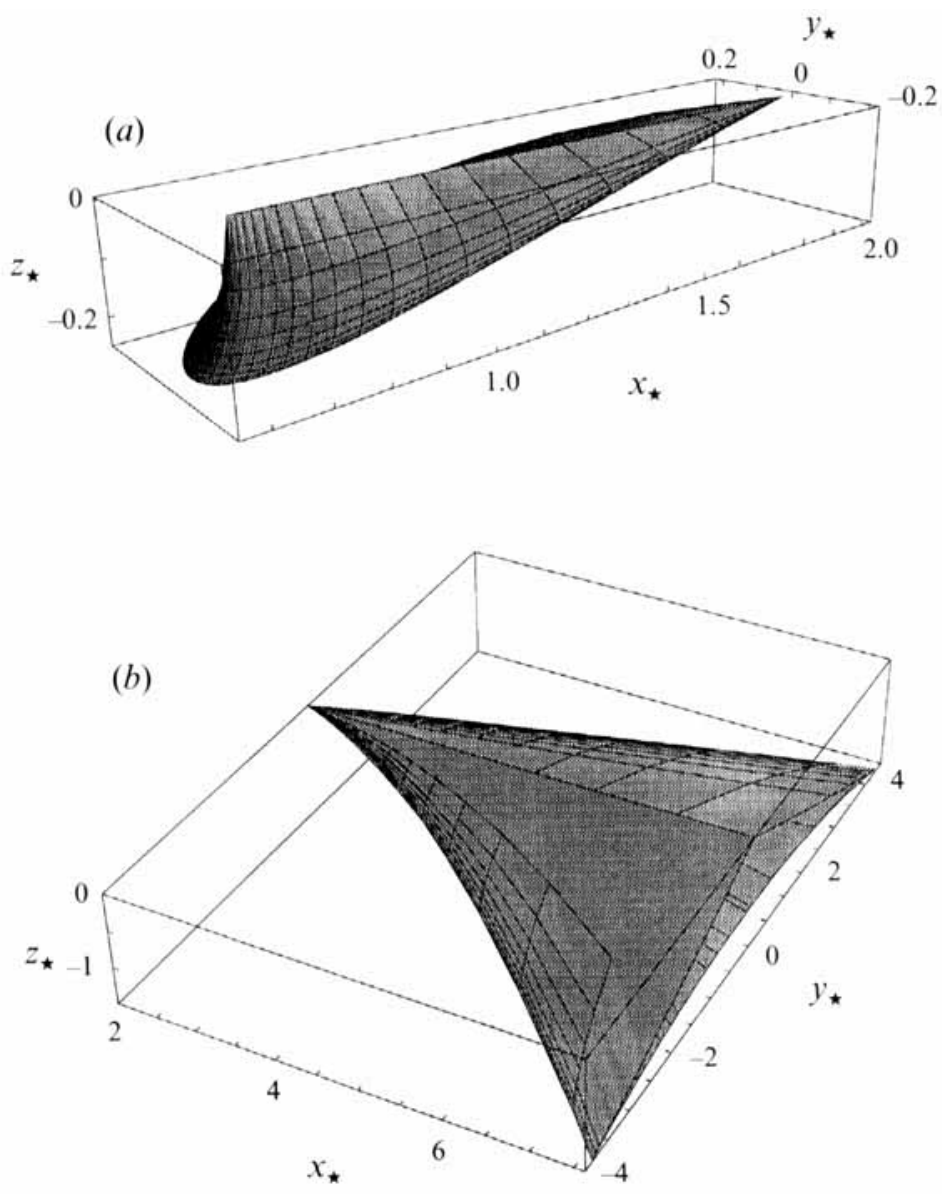

(c)

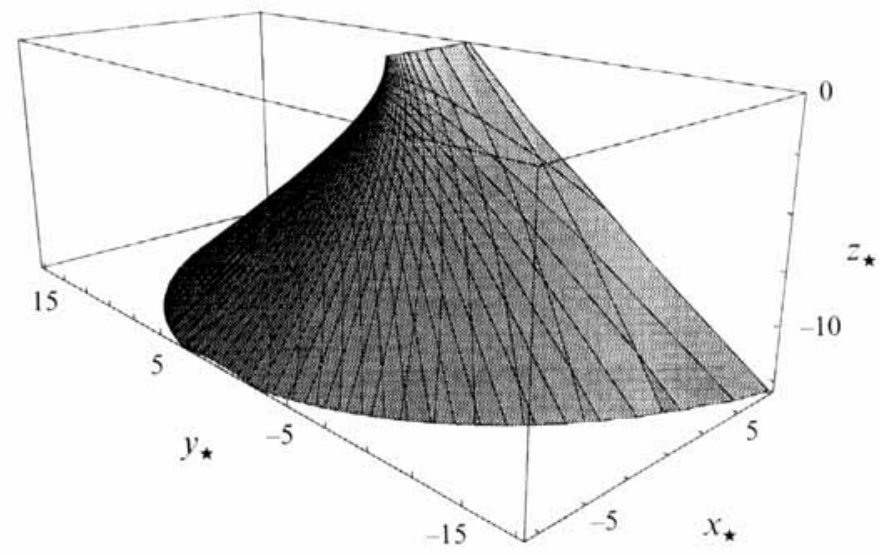

FIGURE 17. Surfaces of constant phase for uniform horizontal motion of an oscillating source, for $r=0.5 ;(a)$ difference waves, $(b)$ downstream sum waves, $(c)$ upstream sum waves. Only the lower half of those surfaces, symmetric about the plane $z_{\star}=0$, is shown; this highlights divergent waves and hides transverse waves. 

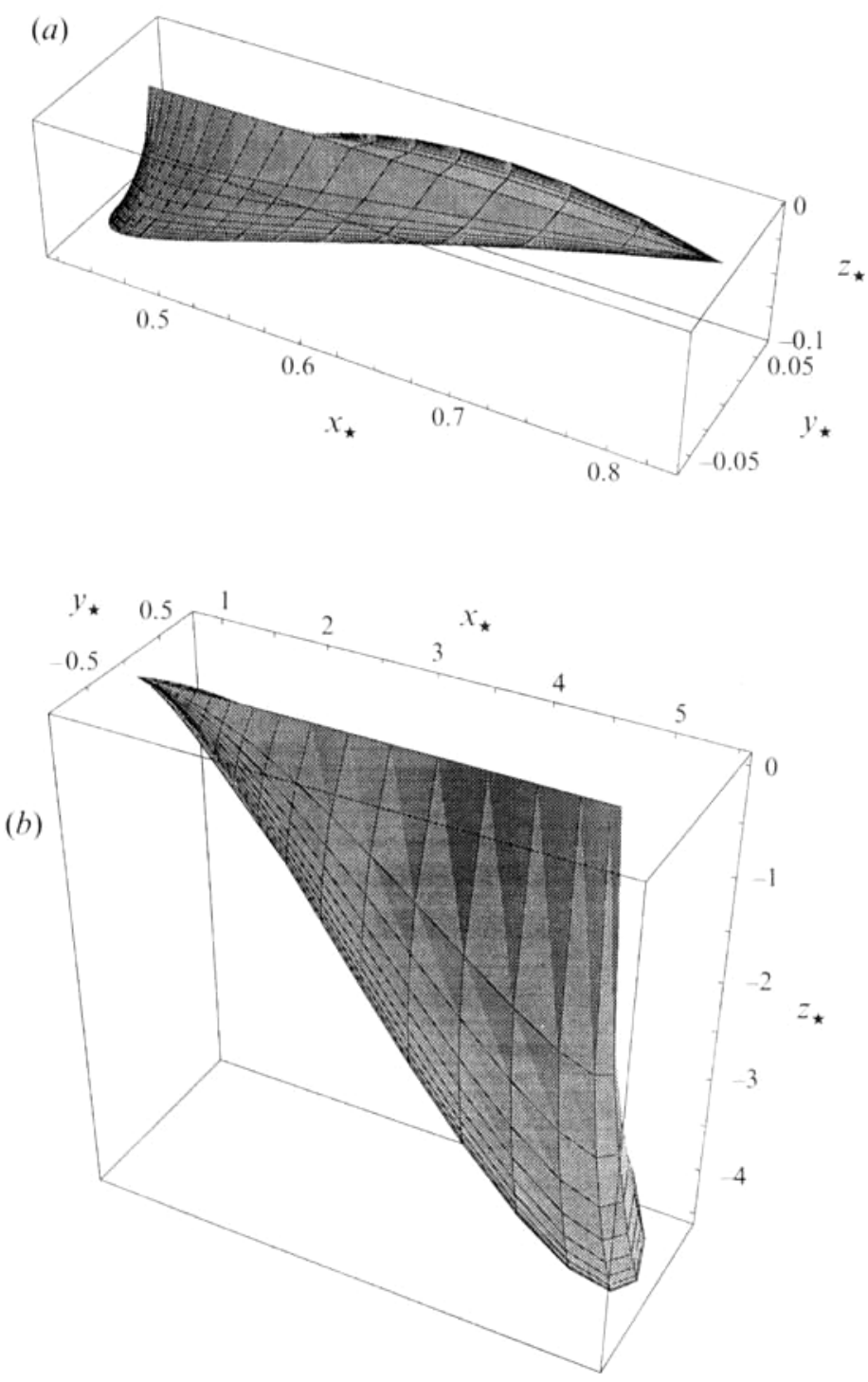

Figure 18. Surfaces of constant phase for uniform horizontal motion of an oscillating source, for $r=1.2 ;(a)$ difference waves, $(b)$ downstream sum waves.

for $N r_{1} / U \gg 1 /\left(\frac{1}{2} \pi-\theta_{1}\right) \gg 1$ or $N r_{\perp} / U \gg N x_{1} / U \gg 1$, with $\left|\sin \varphi_{1}\right|=\Upsilon$,

$$
\xi_{+} \sim r-\frac{2}{9} \Upsilon\left(\frac{\pi}{2}-\theta_{1}\right)^{2} \sim r-\frac{2}{9} r\left(\frac{x_{1}}{r_{\perp}}\right)^{2}
$$

and

$$
\boldsymbol{v}_{+}(\boldsymbol{r}, t) \sim \mathrm{i} H\left(x_{1}\right) \frac{\left(N^{2}-\omega_{0}^{2}\right)^{\frac{1}{2}} m_{0}}{4 \pi \sqrt{ } 3 U r_{\perp}} \frac{\boldsymbol{r}_{\perp}}{r_{\perp}} \exp \left[\mathrm{i}\left(\omega_{0} t-\frac{2}{27} \frac{\omega_{0}}{U} \frac{x_{1}^{3}}{r_{\perp}^{2}}\right)\right] .
$$

All four limiting forms are shown in figures 13,19 and 20. 

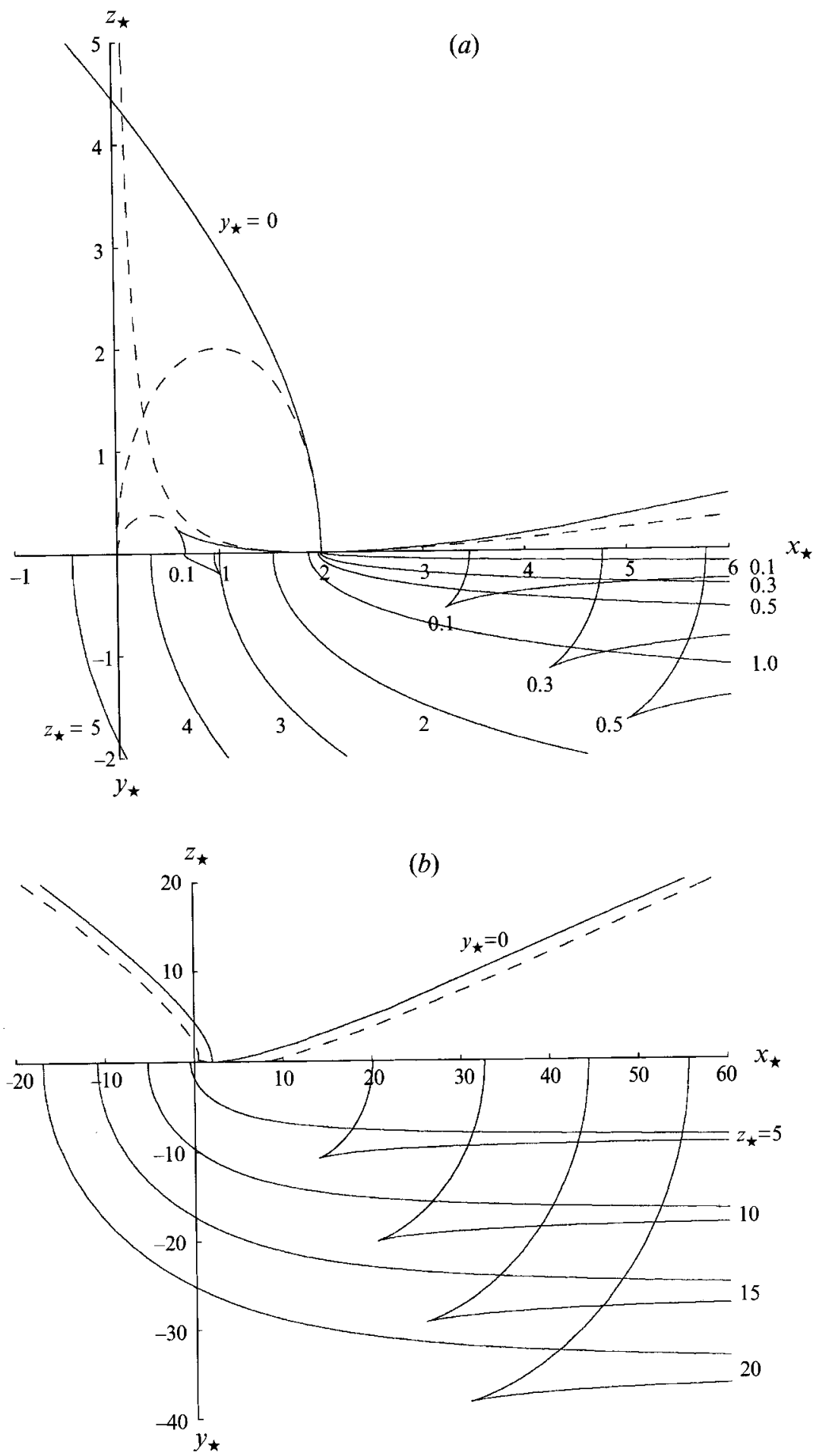

Figure 19. Curves of constant phase for uniform horizontal motion of an oscillating source, for $Y=0.5$. The curves, symmetric about the $x_{\star}$-axis, are drawn above and below it in planes $y_{\star}=0$ and $z_{\star}=$ const, respectively. (a) Moderate $r_{\star}$ : - - exact curves and ---, asymptotic curves $4 Y_{\star}\left(\left|z_{\star}\right|+\frac{1}{2}\right)-Y^{2} x_{\star}^{2} \sim 1$ and $\left(2|Y \mp 1| x_{\star}-1\right)^{2} \mp 2(Y \mp 1)^{3} z_{\star}^{2} \sim 1$, deduced from (7.20) and (7.22) for $y_{\star}=0 ;(b)$ large $r_{\star}$ : 一, exact curves and - - , asymptotic curves $r_{\star}\left(\Theta_{+}-\theta_{1}\right)^{2} \sim 4 \Upsilon /\left(1-\Upsilon^{2}\right)^{\frac{1}{2}}$, deduced from (7.24) for $y_{\star}=0$. 


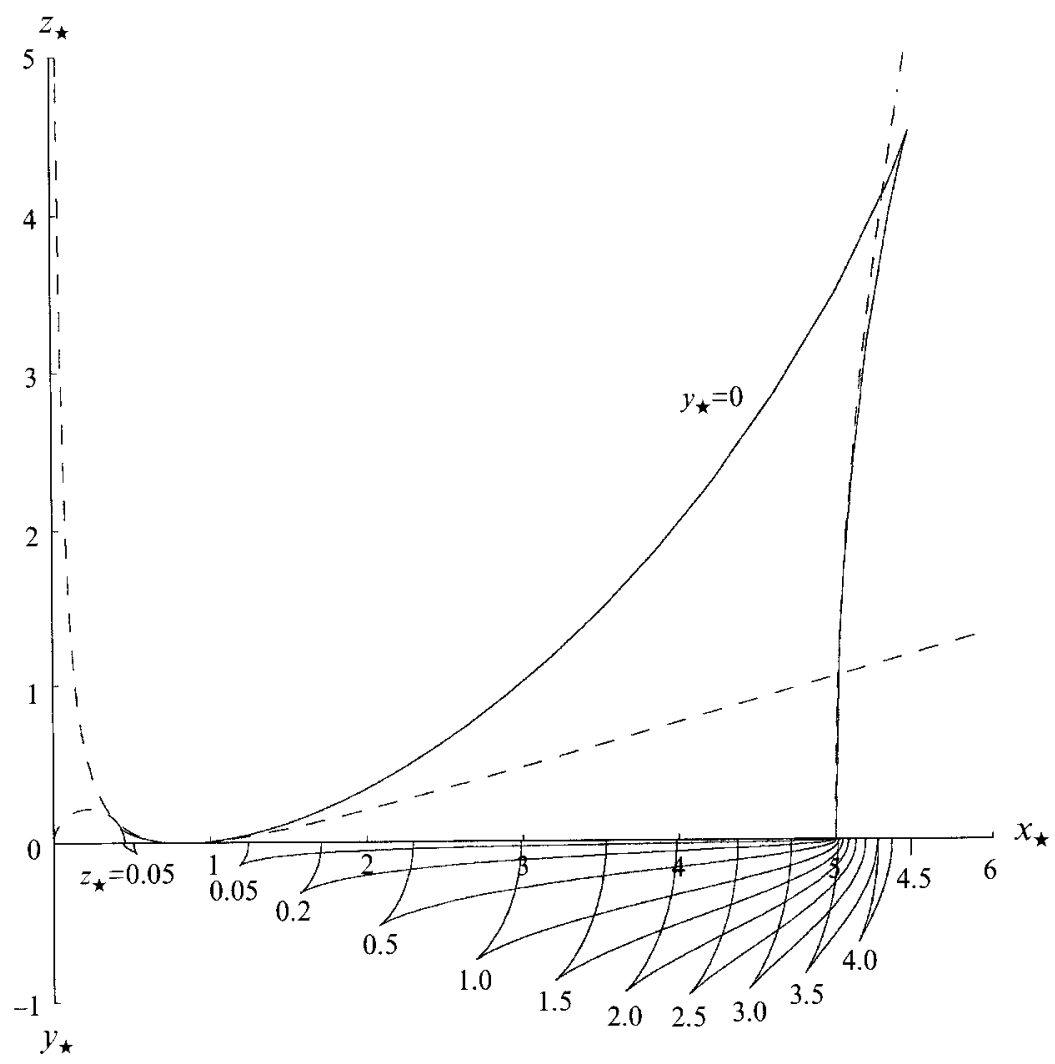

FIGURE 20. Curves of constant phase for uniform horizontal motion of an oscillating source, for $r=1.2$.

\section{Conclusions and discussion}

This paper has applied the Green's function formalism to the problem of internal wave generation by a moving point source. Joint use of the asymptotic form of the impulsive Green's function and of the principle of stationary phase has led to an analysis of the wave field as a collection of impulses radiated by the source all along its path. At each point and time one impulse is received, which was emitted at the retarded time $\tau_{s}$ and has since propagated with the group velocity. An implicit equation defines $\tau_{s}$, in terms of which all characteristics of the waves are determined as characteristics of the associated impulse; differences, attributable to the motion of the source, being the Doppler relation between wavenumbers and frequency, and the modified amplitude. For sources of oscillating strength, moreover, two systems of waves are observed.

For uniform motion an alternative point of view is to consider steady-state waves at the outset. In this way, basic formulae of Lighthill's method and of the 'integral' method mentioned in $\$ 2.1$ are recovered as integrals involving Fourier transforms of the monochromatic Green's function.

Both approaches have been applied to uniform horizontal or vertical motion of a steady source, and to uniform horizontal motion of an oscillatory source. In all three cases explicit expressions of the waves have been found, complementing existing results when there are some. For horizontal motion of a steady source, moreover, two features have been noted, which at first sight can appear as insufficiencies of the 
analysis but which upon careful examination do not compromise its validity. These are as follows.

1. The permanent wave field comprises not only the gravity waves predicted in $\S 3$, but also buoyancy oscillations and a columnar disturbance. In fact, although the approach of $\S 3$ takes all components of the wave field into account, the way that it has been implemented does not; namely, $\S 3$ was aimed at calculating the dominant gravity waves for any motion of a source of any time dependence, not at dealing with contributions insignificant for a point source and of which no general expression can be found. The origin of these contributions is, however, clear, as are the lines along which the analysis could be amended: buoyancy oscillations correspond to the second terms of the integrals (3.8) and (3.24) obtained before the method of stationary phase was applied, while the columnar disturbance is continuously built up by the successive arrivals of waves of vanishing frequency (Bretherton 1967, p. 558), invalidating the use of expansion (3.7) of the Green's function in (3.6).

2. The wave amplitude diverges on the source path, implying infinite wave drag (Gorodtsov \& Teodorovich 1980), and decreases as the source velocity increases, while experiments exhibit some maximum (Makarov \& Chashechkin 1981; Castro et al. 1983). The reason for this lies in the way that real moving bodies have been modelled. As discussed in $\S 2.2$, the same criterion $a \ll U / N$, with $a$ the body scale length and $U / N$ the stratification scale length, justifies classically the use of (i) the same model as in homogeneous flow and (ii) the point source model. For internal waves, however, the adequate scale length is the wavelength $\lambda$, and the correct justification of the point source model is the compactness condition $a \ll \lambda$, which, whatever $U / N a$, cannot be satisfied near the source path where $\lambda \rightarrow 0$. On these grounds, for $U / N a \gg 1$, removing approximation (ii) but maintaining approximation (i), Gorodtsov \& Teodorovich (1982) have proposed the use of the distribution of sources and sinks exactly equivalent to the body in homogeneous flow. Extension of the analysis to such cases is considered in Voisin (1991a).

This work, based on a Ph.D. thesis prepared at Thomson-Sintra Activités SousMarines and Université Pierre et Marie Curie, has been written and supplemented at the Institut de Mécanique de Grenoble. Professor D. G. Crighton is thanked for illuminating discussions at early stages of the study; Professor E. J. Hopfinger and Drs J.-M. Chomaz and P. Bonneton are thanked for stimulating conversations later on. The author is indebted to his research student P. Dupont for fruitful questioning, and for help in computing figures 17-20. Partial financial support by the ANRT through Convention CIFRE No. 249/87 is acknowledged.

\section{Appendix A. Some inverse Fourier transforms}

The following transforms $F(\omega)$ and original functions $f(t)$ have been used:

$$
\begin{array}{cc}
F(\omega) & f(t) \\
\omega^{-\frac{1}{2}} & \mathrm{i} \frac{H(t)}{(\pi t)^{\frac{1}{2}}} \\
K_{0}\left(\alpha \omega^{\frac{1}{2}}\right) & \frac{H(t)}{2 t} \exp \left(\mathrm{i} \frac{\alpha^{2}}{4 t}\right) \\
H(\omega) & \frac{\delta(t)}{2}+\frac{\mathrm{i}}{2 \pi \mathrm{t}} .
\end{array}
$$


They were calculated as in I. Consistently with (4.2), Fourier transformation with respect to time was defined by

$$
\begin{gathered}
F(\omega)=\int f(t) \mathrm{e}^{-\mathrm{i} \omega t} \mathrm{~d} t \equiv \mathrm{FT}[f(t)], \\
f(t)=\frac{1}{2 \pi} f F(\omega) \mathrm{e}^{\mathrm{i} \omega t} \mathrm{~d} \omega \equiv \mathrm{FT}^{-1}[F(\omega)],
\end{gathered}
$$

where the sign - indicates indentation of the integration path below the real singularities of the integrand.

\section{Appendix B. Unsteady evaluation of buoyancy oscillations}

For a steady source in uniform horizontal motion, in the limit $t \rightarrow \infty$ with $\boldsymbol{r}_{1}$ fixed, the buoyancy oscillations appearing in (3.8) become

$$
\psi_{b}(\boldsymbol{r}, t) \sim-\frac{m_{0}}{(2 U)^{\frac{1}{2}}(\pi N)^{\frac{3}{2}}} \int_{0}^{\infty} \frac{\sin \left(\frac{N}{U} \alpha^{2}-\frac{\pi}{4}\right)}{\left[\left(\alpha^{2}-x_{1}\right)^{2}+y^{2}\right]^{\frac{1}{2}}} \mathrm{~d} \alpha,
$$

with $\alpha=[U(t-\tau)]^{\frac{1}{2}}$. Upstream of the source, the inverse square root in the integrand of (B 1) is for $\left|x_{1}\right| \gg|y|$ equivalent to $\left(\alpha^{2}+\left|x_{1}\right|\right)^{-1}$, so that

$$
\psi_{b}(r, t) \sim-\frac{m_{0}}{(2 U)^{\frac{1}{2}}(\pi N)^{\frac{3}{2}}} \int_{0}^{\infty} \frac{\sin \left(\frac{N}{U} \alpha^{2}-\frac{\pi}{4}\right)}{\alpha^{2}+\left|x_{1}\right|} \mathrm{d} \alpha \quad\left(x_{1}<0\right) .
$$

This can be calculated exactly in terms of Fresnel integrals and trigonometric functions, and for $N\left|x_{1}\right| / U \gg 1$ is asymptotic to

$$
\psi_{b}(\boldsymbol{r}, t) \sim \frac{m_{0}}{2^{\frac{5}{2}} \pi N U}\left(\frac{U}{N\left|x_{1}\right|}\right)^{2} \quad\left(x_{1}<0\right) .
$$

Downstream of the source, the inverse square root in the integrand of (B 1) is a maximum $|y|^{-1}$ at $\alpha=x_{1}^{\frac{1}{2}}$, and this maximum is all the more peaked as $x_{1}$ is larger than $|y|$; hence, for $N x_{1} / U \gg 1$, the dominant contribution to (B 1) arises for $x_{1} \gg|y|$ from the vicinity of $x_{1}^{\frac{1}{2}}$. Writing $\alpha=x_{1}^{\frac{1}{2}}+\beta /\left(2 x_{1}^{\frac{1}{2}}\right)$, with $\beta \ll x_{1}$, we obtain

$$
\psi_{b}(\boldsymbol{r}, t) \sim-\frac{m_{0}}{2 \pi N U}\left(\frac{U}{2 \pi N x_{1}}\right)^{\frac{1}{2}} \int_{-\infty}^{\infty} \frac{\sin \left(\frac{N}{U} \beta+\frac{N}{U} x_{1}-\frac{\pi}{4}\right)}{\left(\beta^{2}+y^{2}\right)^{\frac{1}{2}}} \mathrm{~d} \beta \quad\left(x_{1}>0\right),
$$

which is readily evaluated as the product of a modified Bessel function and a trigonometric function, namely

$$
\psi_{b}(\boldsymbol{r}, t) \sim-\frac{m_{0}}{\pi N U}\left(\frac{U}{2 \pi N x_{1}}\right)^{\frac{1}{2}} K_{0}\left(\frac{N}{U}|y|\right) \sin \left(\frac{N}{U} x_{1}-\frac{\pi}{4}\right) \quad\left(x_{1}>0\right) .
$$

Thus, to leading order, for $N t \gg 1, N\left|x_{1}\right| / U \gg 1$ and $\left|x_{1}\right| \gg|y|$, the buoyancy oscillations derived in (3.8) by an unsteady analysis reduce to precisely those derived in (5.23) by a steady analysis. 


\section{REFERENCES}

Aksenov, A. V., Mozhaev, V. V., Skorovarov, V. E. \& Sheronov, A. A. 1989 Stratified flow over a cylinder at low values of the internal Froude number. Fluid Dyn. 24, 639-642.

BaINeS, P. G. 1987 Upstream blocking and airflow over mountains. Ann. Rev. Fluid Mech. 19, $75-97$.

Baines, P. G. \& Grimshaw, R. H. J. 1979 Stratified flow over finite obstacles with weak stratification. Geophys. Astrophys. Fluid Dyn. 13, 317-334.

Baines, P. G. \& HoINKA, K. P. 1985 Stratified flow over two-dimensional topography in fluid of infinite depth: a laboratory simulation. J. Atmos. Sci. 42, 1614-1630.

Belotserkovski, O. M., BelotserkovskiI, S. O., Gushchin, V. A., Morozov, E. N., Onufriev, A. T. \& UL'YANOV, S. A. 1984 Numerical and experimental modeling of internal gravity waves during the motion of a body in a stratified liquid. Sov. Phys. Dokl. 29, 884-886.

BLEISTEIN, N. 1966 Uniform asymptotic expansions of integrals with stationary point near algebraic singularity. Comm. Pure Appl. Maths 19, 353-370.

BleisteIn, N. 1984 Mathematical Methods for Wave Phenomena. Academic.

BLumen, W. \& MCGREgor, C. D. 1976 Wave drag by three-dimensional mountain lee-waves in nonplanar shear flow. Tellus 28, 287-298.

Bonneton, P., Chomaz, J.-M. \& Hopfinger, E. J. 1993 Internal waves produced by the turbulent wake of a sphere moving horizontally in a stratified fluid. J. Fluid Mech. 254, 23-40.

Boyer, D. L., Davies, P. A., Fernando, H. J. S. \& Zhang, X. 1989 Linearly stratified flow past a horizontal circular cylinder. Phil. Trans. R. Soc. Lond. A 328, 501-528.

Bretherton, F. P. 1967 The time-dependent motion due to a cylinder moving in an unbounded rotating or stratified fluid. J. Fluid Mech. 28, 545-570.

BRIGHTon, P. W. M. 1978 Strongly stratified flow past three-dimensional obstacles. Q. J. R. Met. Soc. 104, 289-307.

CASTRO, I. P. 1987 A note on lee wave structures in stratified flow over three-dimensional obstacles. Tellus A 39, 72-81.

Castro, I. P. \& Snyder, W. H. 1988 Upstream motions in stratified flow. J. Fluid Mech. 187, $487-506$.

Castro, I. P., SNyder, W. H. \& Marsh, G. L. 1983 Stratified flow over three-dimensional ridges. J. Fluid Mech. 135, 261-282.

Chashechin, YU. D. 1989 Hydrodynamics of a sphere in a stratified fluid. Fluid Dyn. 24, 1-7.

Chashechin, Yu. D. \& MaKarov, S. A. 1984 Time-varying internal waves. Dokl. Earth Sci. Sect. 276, 210-213.

Cheng, H. K., Hefazi, H. \& Brown, S. N. 1984 Topographically generated cyclonic disturbance and lee waves in a stratified rotating fluid. J. Fluid Mech. 141, 431-453.

Chomaz, J.-M., Bonneton, P. \& Hopfinger, E. J. 1993 The structure of the near wake of a sphere moving horizontally in a stratified fluid. J. Fluid Mech. 254, 1-21.

Clark, T. L. \& Peltier, W. R. 1977 On the evolution and stability of finite-amplitude mountain waves. J. Atmos. Sci. 34, 1715-1730.

Crapper, G. D. 1959 A three-dimensional solution for waves in the lee of mountains. J. Fluid Mech. 6, 51-76.

Crapper, G. D. 1962 Waves in the lee of a mountain with elliptical contours. Phil. Trans. R. Soc. Lond. A 254, 601-623.

Crighton, D. G. \& Oswell, J. E. 1991 Fluid loading with mean flow. I. Response of an elastic plate to localized excitation. Phil. Trans. R. Soc. Lond. A 335, 557-592.

DokuChaEv, V. P. \& Dolina, I. S. 1977 Radiation of internal waves by sources in an exponentially stratified fluid. Izv. Atmos. Ocean. Phys. 13, 444-449.

Drazin, P. G. 1961 On the steady flow of a fluid of variable density past an obstacle. Tellus 13, 239-251.

Ekman, V. W. 1904 On dead water. In Norwegian North Polar Expedition, 1893-1896, Scientific Results (ed. F. Nansen), 5(15). Longmans (1906).

FoldviK, A. \& Wurtele, M. G. 1967 The computation of the transient gravity wave. Geophys. $J$. R. Astron. Soc. 13, 167-185.

GÄRTNER, U. 1983a A note on the visualization and measurement of the internal wave field behind a cylinder moving through a stratified fluid. Geophys. Astrophys. Fluid Dyn. 26, 139-145. 
GärTNER, U. 1983b Visualization of particle displacement and flow in stratified salt water. Exp. Fluids 1, 55-56.

Gärtner, U., Wernekinck, U. \& Merzkirch, W. 1986 Velocity measurements in the field of an internal gravity wave by means of speckle photography. Exp. Fluids 4, 283-287.

GilREATH, H. E. \& BRANDT, A. 1985 Experiments on the generation of internal waves in a stratified fluid. AIAA J. 23, 693-700.

GORODTSOV, V. A. 1980 Radiation of internal waves during vertical motion of a body through a nonuniform liquid. J. Engng Phys. 39, 1062-1065.

GoRODTSOV, V. A. 1981 Radiation of internal waves by rapidly moving sources in an exponentially stratified liquid. Sov. Phys. Dokl. 26, 229-230.

Gorodtsov, V. A. $1991 \mathrm{High}$-speed asymptotic form of the wave resistance of bodies in a uniformly stratified liquid. J. Appl. Mech. Tech. Phys. 32, 331-337.

Gorodtsov, V. A. \& TeODORovich, E. V. 1980 On the generation of internal waves in the presence of uniform straight-line motion of local and nonlocal sources. Izv. Atmos. Ocean. Phys. 16, 699-704.

Gorodtsov, V. A. \& Teodorovich, E. V. 1981 Two-dimensional problem for internal waves generated by moving singular sources. Fluid Dyn. 16, 219-224.

Gorodtsov, V. A. \& Teodorovich, E. V. 1982 Study of internal waves in the case of rapid horizontal motion of cylinders and spheres. Fluid Dyn. 17, 893-898.

Gorodtsov, V. A. \& TEODorovich, E. V. 1983 Radiation of internal waves by periodically moving sources. J. Appl. Mech. Tech. Phys. 24, 521-526.

Gradshteyn, I. S. \& Ryzhik, I. M. 1980 Tables of Integrals, Series, and Products. Academic.

Grigor'Ev, G. I. \& Dokuchaev, V. P. 1970 On the theory of the radiation of acoustic-gravity waves by mass sources in a stratified isothermal atmosphere. Izv. Atmos. Ocean. Phys. 6, 398-402.

Grimshaw, R. H. J. 1969 Slow time-dependent motion of a hemisphere in a stratified fluid. Mathematika 16, 231-248.

Hanazaki, H. 1988 A numerical study of three-dimensional stratified flow past a sphere. J. Fluid Mech. 192, 393-419.

HANAZAKI, H. 1989 Drag coefficient and upstream influence in three-dimensional stratified flow of finite depth. Fluid Dyn. Res. 4, 317-332.

Haussling, H. J. 1977 Viscous flows of stably stratified fluids over barriers. J. Atmos. Sci. 34, $589-602$.

Hawthorne, W. R. \& Martin, M. E. 1955 The effect of density gradient and shear on the flow over a hemisphere. Proc. R. Soc. Lond. A 232, 184-195.

Hefazi, H. T. \& ChenG, H. K. 1988 The evolution of cyclonic disturbances and lee waves over a topography in a rapidly rotating stratified flow. J. Fluid Mech. 195, 57-76.

Hopfinger, E. J., Flor, J.-B., Chomaz, J.-M. \& Bonneton, P. 1991 Internal waves generated by a moving sphere and its wake in a stratified fluid. Exp. Fluids 11, 255-261.

Hunt, J. C. R. \& SNyder, W. H. 1980 Experiments on stably and neutrally stratified flow over a model three-dimensional hill. J. Fluid Mech. 96, 671-704.

JANOWITZ, G. S. 1984 Lee waves in three-dimensional stratified flow. J. Fluid Mech. 148, 97-108.

KLEMP, J. B. \& LILLY, D. K. 1978 Numerical simulation of hydrostatic mountain waves. J. Atmos. Sci. 35, 78-107.

Landau, L. \& LifChitz, E. 1970 Théorie des Champs. Mir.

Lighthill, M. J. 1958 Introduction to Fourier Analysis and Generalised Functions. Cambridge University Press.

LighthiLl, M. J. 1967 On waves generated in dispersive systems by travelling forcing effects, with applications to the dynamics of rotating fluids. J. Fluid Mech. 27, 725-752.

Lighthill, M. J. 1978 Waves in Fluids. Cambridge University Press.

Lighthill, M. J. 1986 An Informal Introduction to Theoretical Fluid Mechanics. Oxford University Press.

Lin, Q., Lindberg, W. R., Boyer, D. L. \& Fernando, H. J. S. 1992 Stratified flow past a sphere. J. Fluid Mech. 240, 315-354.

Makarov, S. A. \& Chashechin, YU. D. 1981 Apparent internal waves in a fluid with exponential density distribution. J. Appl. Mech. Tech. Phys. 22, 772-779. 
Makarov, S. A. \& ChaSheChKin, YU. D. 1982 Coupled internal waves in a viscous incompressible fluid. Izv. Atmos. Ocean. Phys. 18, 758-764.

Miles, J. W. $1969 a$ The lee-wave régime for a slender body in a rotating flow. J. Fluid Mech. 36, 265-288.

Miles, J. W. $1969 b$ Transient motion of a dipole in a rotating flow. J. Fluid Mech. 39, 433-442.

MiLes, J. W. $1969 c$ Waves and wave drag in stratified flows. In Proc. XIIth International Congress of Applied Mechanics (ed. M. Hétényi \& W. G. Vincenti), pp. 50-76. Springer.

MiLES, J. W. 1971 Internal waves generated by a horizontally moving source. Geophys. Fluid Dyn. 2, 63-87.

Miles, J. W. \& Huppert, H. E. 1969 Lee waves in a stratified flow. Part 4. Perturbation approximations. J. Fluid Mech. 35, 497-525.

Morse, P. M. \& Feshbach, H. 1953 Methods of Theoretical Physics. Part I. McGraw-Hill.

Mowbray, D. E. \& RaRITY, B. S. H. 1967 The internal wave pattern produced by a sphere moving vertically in a density stratified liquid. J. Fluid Mech. 30, 489-495.

Murdock, J. W. 1977 The near-field disturbance created by a body in a stratified medium with a free surface. Trans. ASME E: J. Appl. Mech. 44, 534-540.

Peat, K. S. \& Stevenson, T. N. 1975 Internal waves around a body moving in a compressible density-stratified fluid. $J$. Fluid Mech. 70, 673-688.

Peat, K. S. \& Stevenson, T. N. 1976 The phase configuration of waves around a body moving in a rotating stratified fluid. J. Fluid Mech. 75, 647-656.

Peltier, W. R. \& Clark, T. L. 1979 The evolution and stability of finite-amplitude mountain waves. Part II: Surface wave drag and severe downslope windstorms. J. Atmos. Sci. 36, 14981529.

Peltier, W. R. \& Clark, T. L. 1983 Nonlinear mountain waves in two and three spatial dimensions. Q. J. R. Met. Soc. 109, 527-548.

Phillips, D. S. 1984 Analytical surface pressure and drag for linear hydrostatic flow over threedimensional elliptical mountains. J. Atmos. Sci. 41, 1073-1084.

REDEKOPP, L. G. 1975 Wave patterns generated by disturbances travelling horizontally in rotating stratified fluids. Geophys. Fluid Dyn. 6, 289-313.

REHM, R. G. \& RADT, H. S. 1975 Internal waves generated by a translating oscillating body. $J$. Fluid Mech. 68, 235-258.

Rotunno, R. \& Smolarkiewicz, P. K. 1991 Further results on lee vortices in low-Froude-number flow. J. Atmos. Sci. 48, 2204-2211.

Sarma, L. V. K. V. \& Krishna, D. V. 1972 Motion of a sphere in a stratified fluid. Zastosow. Matem. $13,123-130$.

SCORER, R. S. 1956 Airflow over an isolated hill. Q. J. R. Met. Soc. 82, 75-81.

Sharman, R. D. \& Wurtele, M. G. 1983 Ship waves and lee waves. J. Atmos. Sci. 40, 396-427.

Sмith, R. B. 1980 Linear theory of stratified hydrostatic flow past an isolated mountain. Tellus 32, 348-364.

SMith, R. B. 1988 Linear theory of stratified flow past an isolated mountain in isosteric coordinates. J. Atmos. Sci. 45, 3889-3896.

Sмith, R. B. 1989 Mountain-induced stagnation points in hydrostatic flow. Tellus A 41, 270-274.

Smolarkiewicz, P. K. \& Rotunno, R. 1989 Low Froude number flow past three-dimensional obstacles. Part I: Baroclinically generated lee vortices. J. Atmos. Sci. 46, 1154-1164.

Stevenson, T. N. 1968 Some two-dimensional internal waves in a stratified fluid. J. Fluid Mech. 33, $715-720$.

Stevenson, T. N. 1969 Axisymmetric internal waves generated by a travelling oscillating body. $J$. Fluid Mech. 35, 219-224.

Stevenson, T. N. 1973 The phase configuration of internal waves around a body moving in a density stratified fluid. J. Fluid Mech. 60, 759-767.

Stevenson, T. N., Chang, W. L. \& Laws, P. 1979 Viscous effects in lee waves. Geophys. Astrophys. Fluid Dyn. 13, 141-151.

Stevenson, T. N. \& Thomas, N. H. 1969 Two-dimensional internal waves generated by a travelling oscillating cylinder. J. Fluid Mech. 36, 505-511.

Stevenson, T. N., Woodhead, T. J. \& Kanellopulos, D. 1983 Viscous effects in some internal waves. Appl. Sci. Res. 40, 185-197. 
STUROVA, I. V. 1974 Wave motions produced in a stratified liquid from flow past a submerged body. J. Appl. Mech. Tech. Phys. 15, 796-805.

STURova, I. V. 1978 Internal waves generated by local disturbances in a linearly stratified liquid of finite depth. J. Appl. Mech. Tech. Phys. 19, 330-336.

STUROVA, I. V. 1980 Internal waves generated in an exponentially stratified fluid by an arbitrarily moving source. Fluid Dyn. 15, 378-383.

Subba RaO, V. \& Prabhakara RaO, G. V. 1971 On waves generated in rotating stratified liquids by travelling forcing effects. J. Fluid Mech. 46, 447-464.

Suzuki, M. \& Kuwahara, K. 1992 Stratified flow past a bell-shaped hill. Fluid Dyn. Res. 9, 1-18.

SYKES, R. I. 1978 Stratification effects in boundary layer flow over hills. Proc. R. Soc. Lond. A 361, 225-243.

Sysoeva, E. Ya. \& Chashechin, Yu. D. 1991 Vortex systems in the stratified wake of a sphere. Fluid Dyn. 26, 544-551.

ThORPE, S. A. 1975 The excitation, dissipation, and interaction of internal waves in the deep ocean. J. Geophys. Res. 80, 328-338.

Trubnikov, B. N. 1959 The three-dimensional problem of the flow over a barrier of an air current unbounded at the top. Dokl. Earth Sci. Sect. 129, 1136-1138.

UMEKI, M. \& KAMBE, T. 1989 Stream patterns of an isothermal atmosphere over an isolated mountain. Fluid Dyn. Res. 5, 91-109.

Vladimirov, V. A. \& IL'in, K. I. 1991 Slow motions of a solid in a continuously stratified fluid. $J$. Appl. Mech. Tech. Phys. 32, 194-200.

VoIsIN, B. 1991 a Rayonnement des ondes internes de gravité. Application aux corps en mouvement. Ph.D. thesis, Universite Pierre et Marie Curie, Paris.

VoIsin, B. $1991 b$ Internal wave generation in uniformly stratified fluids. Part 1. Green's function and point sources. $J$. Fluid Mech. 231, 439-480 (referred to herein as I).

Warren, F. W. G. 1960 Wave resistance to vertical motion in a stratified fluid. J. Fluid Mech. 7, 209-229.

WOODHEAD, T. J. 1983 The phase configuration of the waves around an accelerating disturbance in a rotating stratified fluid. Wave Motion 5, 157-165.

Wu, T. Y.-T. 1965 Three-dimensional internal gravity waves in a stratified free-surface flow. $Z$. Angew. Math. Mech. Sond. 45, T194-T195.

Wurtele, M. G. 1957 The three-dimensional lee wave. Beitr. Phys. Atmos. 29, 242-252. 